\title{
Enriched Cd141+ DCs in the joint are transcriptionally distinct, activated, and contribute to joint pathogenesis
}

\author{
Mary Canavan, ${ }^{1}$ Alice M. Walsh, ${ }^{2}$ Vipul Bhargava, ${ }^{3}$ Sarah M. Wade, ${ }^{1}$ Trudy McGarry, ${ }^{1}$ \\ Viviana Marzaioli, ${ }^{1}$ Barry Moran, ${ }^{4}$ Monika Biniecka, ${ }^{5}$ Hannah Convery, ${ }^{5}$ Siobhan Wade, ${ }^{1}$ Carl Orr, ${ }^{5}$ \\ Ronan Mullan, ${ }^{6}$ Jean M. Fletcher, ${ }^{7}$ Sunil Nagpal, ${ }^{2}$ Douglas J. Veale, ${ }^{5}$ and Ursula Fearon ${ }^{1}$ \\ ${ }^{1}$ Molecular Rheumatology, School of Medicine, Trinity College Dublin, Ireland. ${ }^{2}$ Immunology and ${ }^{3}$ Discovery Sciences, \\ Janssen Research \& Development, Spring House, Pennsylvania, USA. ${ }^{4}$ School of Biochemistry and Immunology, Trinity \\ College Dublin, Ireland. ${ }^{5}$ Centre for Arthritis \& Rheumatic Diseases, St. Vincent's University Hospital, University College \\ Dublin, Ireland. 'Department of Rheumatology, Adelaide and Meath Hospital, Dublin, Ireland. ${ }^{7}$ Translational Immunology, \\ Schools of Biochemistry and Immunology and Medicine, Trinity College Dublin, Ireland.
}

CD141+ DC are implicated in antiviral and antitumor immunity. However, mechanistic studies in autoimmune disease are limited. This is the first study to our knowledge examining CD141 ${ }^{+}$DC in autoimmune disease, specifically inflammatory arthritis (IA). We identified significant enrichment of CD141 ${ }^{+} \mathrm{DC}$ in the inflamed synovial joint, which were transcriptionally distinct from IA and healthy control (HC) blood CD141+ DC and significantly more activated, and they exhibited increased responsiveness to TLR3. Synovial CD141+ DC represent a bone fide CD141+ DC population that is distinct from CD1c $\mathrm{DC}$. Synovial CD141+ DC induced higher levels of CD4 ${ }^{+}$and CD8 ${ }^{+} \mathrm{T}$ cell activation compared with their peripheral blood counterparts, as made evident by expression of IFN- $\gamma$, TNF- $\alpha$, and granulocyte-macrophage CSF (CMCSF). Autologous synovial CD141+ DC cocultures also induce higher levels of these cytokines, further highlighting their contribution to synovial inflammation. Synovial CD141+ DC-T cell interactions had the ability to further activate synovial fibroblasts, inducing adhesive and invasive pathogenic mechanisms. Furthermore, we identify a mechanism in which synovial CD141+ DC are activated, via ligation of the hypoxia-inducible immune-amplification receptor TREM-1, which increased synovial CD141+ DC activation, migratory capacity, and proinflammatory cytokines. Thus, synovial CD141+ DC display unique mechanistic and transcriptomic signatures, which are distinguishable from blood CD141+ DC and can contribute to synovial joint inflammation.

Conflict of interest: The authors have declared that no conflict of interest exists.

License: Copyright 2018, American Society for Clinical Investigation.

Submitted: May 26, 2017

Accepted: October 29, 2018

Published: December 6, 2018

Reference information: JCI Insight. 2018;3(23):e95228. https://doi.org/10.1172/jci. insight.95228.

\section{Introduction}

DC are central players in the control of innate and adaptive immune responses. Due to their rarity, difficulty in isolating, and lack of definitive markers, our understanding of human DC biology, especially in disease, is limited. Studies to date have identified 2 distinct populations of human peripheral blood (PB) DC - myeloid DC $(\mathrm{mDC})$ and plasmacytoid DC (pDC). pDC are involved in antiviral immunity and produce large amounts of type I IFNs, whereas $\mathrm{mDC}$ have potent antigen presenting abilities and are strong inducers of $\mathrm{CD}^{+}$and $\mathrm{CD} 8^{+}$ $\mathrm{T}$ cell responses. Myeloid or conventional DC can be further subdivided into the $\mathrm{CD} 1 \mathrm{c}^{+} \mathrm{DC}$ and the more newly described CD141+ $\left(\mathrm{BDCA}-3^{+}\right) \mathrm{DC}$. The functional properties of CD $141^{+} \mathrm{DC}$ and their functional homology to mouse $\mathrm{CD}^{+} \mathrm{DC}$ were described in $2010(1-3)$. As a consequence of these studies, our understanding of $\mathrm{PB}$ $\mathrm{CD} 141^{+} \mathrm{DC}$ has vastly improved. CD $141^{+} \mathrm{DC}$ are superior at cross-presentation of exogenous antigen, in addition to necrotic or dead cell antigens, and produce high levels of type III IFNs and have the capacity to produce type I IFNs and IL-12p70 (1-4). They have high expression of TLR3, Clec9A, and the chemokine receptor XCR-1 $(1,2)$. CD $141^{+}$DC represent an extremely rare subset of PB DC comprising approximately $0.03 \%$ of $\mathrm{PB}$ mononuclear cell (PBMC). The majority of studies to date on human CD141 ${ }^{+} \mathrm{DC}$ have been performed in $\mathrm{PB}$; however, these specialized DC have also been identified in tonsil, BM, spleen, liver, lung, and skin $(3,5-8)$ Inflammatory arthritis (IA) - which includes rheumatoid arthritis (RA) and psoriatic arthritis (PsA) — is a chronic autoimmune disease that principally affects the joints. Synovial fibroblast proliferation, 
increased angiogenesis, and leucocyte infiltration transforms the synovial membrane into an aggressive tumor-like pannus. Subsequently, this facilitates the invasion of synovial fibroblasts into adjacent cartilage and bone, resulting in joint destruction and functional disability. While previous studies have identified a potential role for DC in the pathogenesis of IA (9-11), CD141 $1^{+}$DC have not been described in IA, to date, or in the context of any of the rheumatic diseases. Indeed, due to their rarity, limited studies exist on the functional role of $\mathrm{CD} 141^{+} \mathrm{DC}$ in autoimmunity. Therefore, the aim of this study was to explore the role of $\mathrm{CD} 141^{+} \mathrm{DC}$ in the pathogenesis of IA, using both functional and transcriptomic analysis, with a specific focus on the function of CD141 ${ }^{+} \mathrm{DC}$ isolated from the site of inflammation (inflamed synovial joint), compared with PB CD $141^{+}$DC. RNA sequencing (RNA-seq) analysis allowed us to examine the diversity of joint $\mathrm{CD} 141^{+} \mathrm{DC}$ compared with their blood counterparts, and this - along with a computational system biology approach - led to identification of key signaling pathways distinct to synovial DC that may provide insight into new therapeutic strategies. In addition, due to the central role that synovial fibroblasts play in tissue destruction and disease progression, we examined the effect of CD $141^{+} \mathrm{DC}$ on synovial fibroblast invasive mechanisms. Furthermore, we identified a mechanism of action by which synovial CD $141^{+}$ DC are activated, via ligation of the hypoxia-inducible immune-amplification receptor triggering receptor expressed on myeloid cells-1 (TREM-1).

\section{Results}

$D C$ are enriched at the site of inflammation, where they reside in a semimature state. The phenotype and frequency of both $\mathrm{mDC}$ and $\mathrm{pDC}$ was assessed in $\mathrm{PB}$ of IA patients and healthy control (HC). DC were identified as Lin HLA-DR ${ }^{+}$and further characterized into CD11 $\mathrm{c}^{+} \mathrm{HLA}-\mathrm{DR}^{+} \mathrm{mDC}$ or $\mathrm{CD} 123^{+} \mathrm{HLA}-\mathrm{DR}^{+} \mathrm{pDC}$. We identified a significant decrease in the percentage of circulating $\mathrm{mDC}$ in IA patients compared with $\mathrm{HC}(P<0.05)$ with no change in the frequency of $\mathrm{pDC}$ (Figure 1A). With further examination of the $\mathrm{mDC}$ subset, we observed a significant decrease in the percentage (Figure $1 \mathrm{~B} ; P<0.05$ ) and absolute number (Supplemental Figure 1 ; supplemental material available online with this article; https://doi.org/10.1172/jci.insight.95228DS1) of circulating $\mathrm{CD}_{141^{+}} \mathrm{DC}$ in IA patients compared with $\mathrm{HC}$ but no significant change in circulating CD1c $\mathrm{c}^{+} \mathrm{DC}$ between the 2 groups. IA mDC showed similar cell surface expression of the DC maturation marker CD40 to that of $\mathrm{HC}$ (Figure 1C). We hypothesized that decreased circulating levels of $\mathrm{mDC}$ in IA could be attributed to increased synovial infiltration. We therefore examined infiltrating DC within IA synovial tissue (ST) compared with circulating DC in patient-matched PB. Analysis demonstrated that in IA ST, CD45 $5^{+} \mathrm{HLA}-\mathrm{DR}{ }^{+} \mathrm{C}-$ $\mathrm{D} 11 \mathrm{c}^{+}$antigen presenting cells (APC) were significantly enriched compared with APC residing in the PB $(P<0.05)$ (Figure 1D). These cells displayed a more mature phenotype with significantly higher levels of CD80 and CD40 compared with DC within the PB (Figure 1E; $P<0.01$ and $P<0.05$, respectively). Furthermore, the percentage of DC within IA synovial fluid (SF) compared with $\mathrm{PB}$ was examined (representative plot, Figure $1 \mathrm{~F}$ ), and a significant enrichment in $\mathrm{CD} 11 \mathrm{c}^{+} \mathrm{mDC}$ was demonstrated in SF mononuclear cells (SFMC) compared with matched PBMC (Figure 1G; $P<0.05$ ). Synovial $\mathrm{mDC}$ have increased expression of the costimulatory marker CD40 (Figure $1 \mathrm{H} ; P<0.05$ ), further highlighting the site-specific differences in DC activation markers. These data suggest that DC are enriched at the site of inflammation, where they reside in a more mature state than PB DC.

$C D 141^{+} D C$ are enriched in inflamed synovium and are distinct from $P B D C$. Our data suggest that DC within the inflamed joint are more activated than their PB counterparts. Immunohistochemical analysis of DC subsets in ST revealed a significant increase in both $\mathrm{CD}_{141}{ }^{+}(P<0.01)$ and $\mathrm{Clec} 9 \mathrm{~A}^{+}(P<0.01)$ cells in RA synovium compared with osteoarthritis (OA) (Supplemental Figure 2, A and B). OA is a noninflammatory form of arthritis, which is not characterized by synovial immune infiltration. In contrast to this, PsA/RA are characterized by extensive synovial inflammation and immune cell infiltration; consequently, OA tissue in this regard acts as a noninflammatory disease comparator. Subsequent immunofluorescence staining of RA ST demonstrated colocalization of CD141 and Clec9A staining (Supplemental Figure 2C and Supplemental Figure 3). CD141 lining layer (LL) and Clec9A sublining layer (SL) expression significantly correlated with macroscopic synovitis ( $r=0.5991$ and $P=0.0396 ; r=0.6541$ and $P=0.0290$, respectively; Supplemental Figure 2E). Representative in vivo images of macroscopic synovitis are shown in Supplemental Figure 2D.

Analysis of IA SF identified a significant enrichment in the CD $141^{+}$DC population compared with matched $\mathrm{PB}(P<0.01$, Figure $2 \mathrm{~A})$. Previous studies have shown that monocyte-derived DC or CD1 $\mathrm{c}^{+} \mathrm{DC}$ express CD141 in the context of inflammation. Therefore, we used multicolor flow cytometry and RNA analysis to verify that these cells were bona fide $\mathrm{CD} 141^{+} \mathrm{DC}$. For flow cytometry experiments, $\mathrm{CD} 141^{+} \mathrm{DC}$ 
A

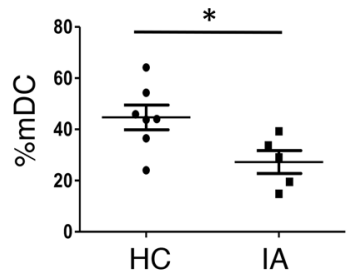

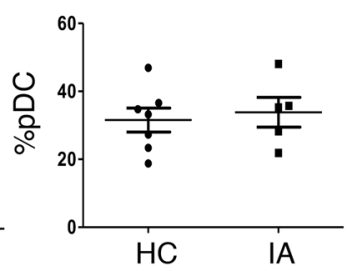

D
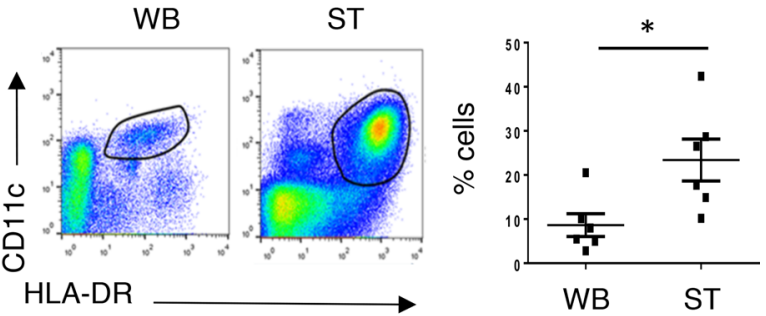

B
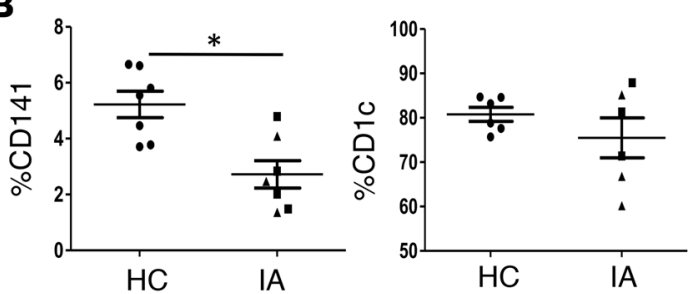

E
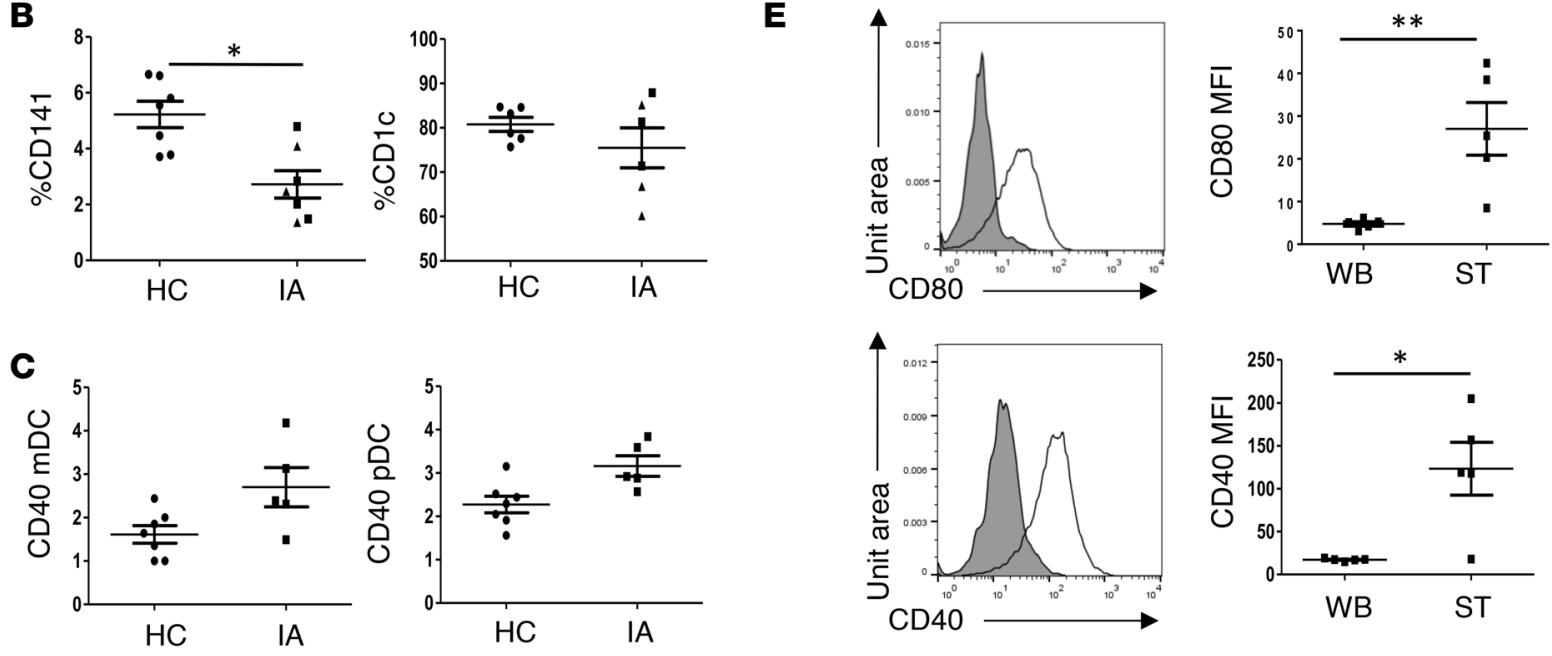

$\mathbf{F}$

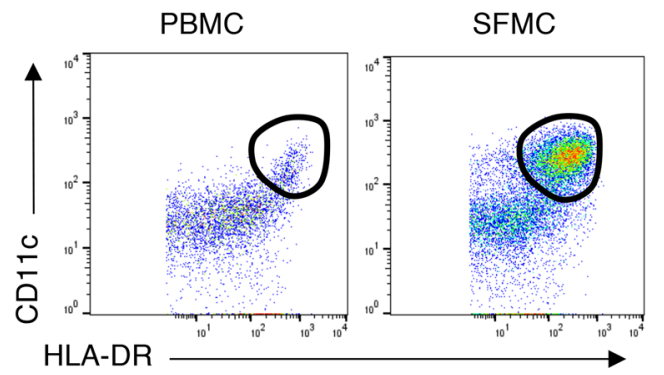

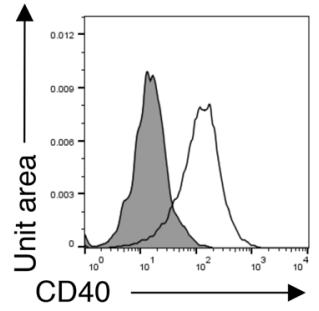

G

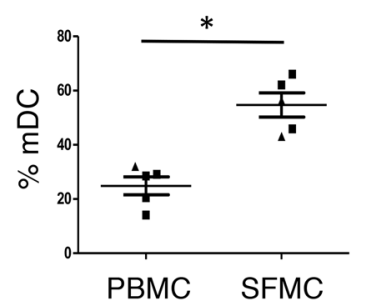

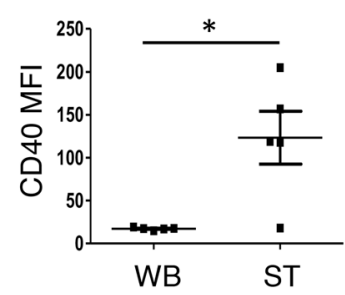

H

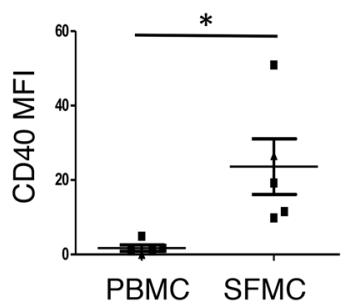

Figure 1. DC are enriched at the site of inflammation, where they reside in a semimature state. (A) Frequency of mDC and pDC in the peripheral blood of healthy control $(\mathrm{HC})$ donors $(n=7)$ and inflammatory arthritis $(\mathrm{IA})$ patients $(n=5)$. Frequency was calculated as percentage of $\mathrm{mDC}$ and $\mathrm{pDC}$ in the Lin-HLADR ${ }^{+}$population. mDC were gated as Lin-HLADR ${ }^{+}$CD11 $c^{+}$cells, and $\mathrm{pDC}$ were gated as $\mathrm{Lin}^{-} \mathrm{HLADR}^{+} \mathrm{CD} 123^{+}$cells. (B) The percentage of CD141 ${ }^{+} \mathrm{mDC}^{-}$and $\mathrm{CD}_{1} \mathrm{c}^{+} \mathrm{mDC}$ was analyzed in $\mathrm{HC}$ and IA patients as a percentage of $\mathrm{Lin}^{-} \mathrm{HLADR}^{+} \mathrm{CD} 11 \mathrm{c}^{+}$cells $(\mathrm{mDC})(n=7)$. $\mathrm{mDC}$ were gated as described above, and CD141+ $\mathrm{DC}$ were gated on $\mathrm{CD} 1 \mathrm{c}^{-} \mathrm{CD} 141^{+} \mathrm{Clec} 9 \mathrm{~A}^{+}$cells. CD1 $\mathrm{c}^{+} \mathrm{mDC}$ were gated based on the expression of $\mathrm{CD} 1 \mathrm{c}$ in the mDC population. (C) The median fluorescence intensity (MFI) of CD40 on mDC and pDC in HC and IA. (D) Identification of DC within the inflamed synovium. Dissociated IA synovial tissue (ST) and matched

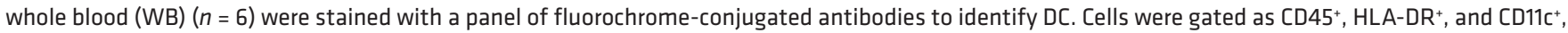
and the MFI of CD80 and CD40 was assessed. Percentage of MDC in ST and WB of IA patients as a percentage of CD45 cells. (E) Representative histogram of CD80 and CD40 expression in WB (shaded area) and ST (clear line) and bar chart representing MFI. (F) Frequency and activation of mDC in synovial fluid and matched peripheral blood of IA patients $(n=5)$. DC were gated as described in A. (C) The percentage of mDC in IA PBMC and IA synovial fluid mononuclear cells (SFMC) is presented as a percentage of Lin-HLA-DR+ cells. (H) The MFI of CD40 on mDC is represented in IA PBMC and SFMC. Data were analyzed using Mann-Whitney $U$ test. ${ }^{*} P<0.05$, ${ }^{* *} P<0.01$ significantly different from control. Square dots represent RA patients; triangles represent PsA patients. IA, inflammatory arthritis; SFMC, synovial fluid mononuclear cells

were characterized as $\mathrm{Lin}^{-} \mathrm{HLA}-\mathrm{DR}{ }^{+} \mathrm{CD} 11 \mathrm{c}^{+} \mathrm{CD} 1 \mathrm{c}^{-} \mathrm{CD} 141^{+} \mathrm{Clec}^{9} \mathrm{~A}^{+}$and $\mathrm{XCR}-1^{+}$(Figure 2B). In addition, the expression of CD141-related genes were examined in $\mathrm{CD} 141^{+} \mathrm{DC}$ from IA blood, SF, and HC blood in comparison with PB CD1 $\mathrm{c}^{+}$DC (Figure 2C). As expected, the transcript levels of XCR-1, TLR3, $\mathrm{CADM}$, and Clec9A were all upregulated in the $\mathrm{CD} 141^{+} \mathrm{DC}$ populations, while $\mathrm{CD} 1 \mathrm{c}$ and $\mathrm{Clec} 10 \mathrm{~A}$ were decreased in the $\mathrm{CD} 141^{+} \mathrm{DC}$ populations compared with $\mathrm{CD} 1 \mathrm{c}^{+} \mathrm{DC}$.

To further demonstrate differences in the phenotype and function of $\mathrm{CD} 141^{+} \mathrm{DC}$ between the $\mathrm{PB}$ and the site of inflammation, RNA-seq analysis was performed. CD141 ${ }^{+}$DC were sorted from IA SF $(n=6)$, 
A

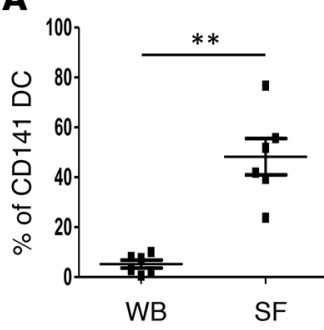

C

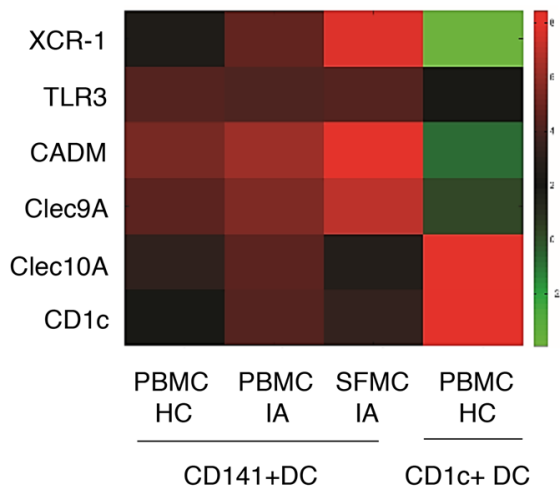

B

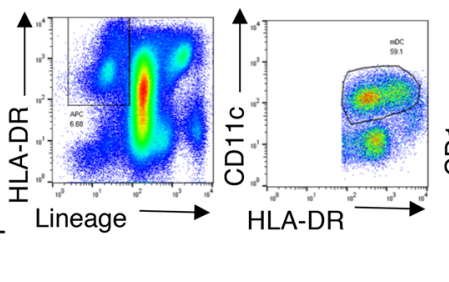

D
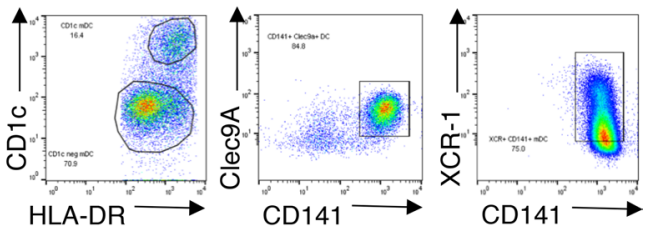

CD141+DC PCA Plots
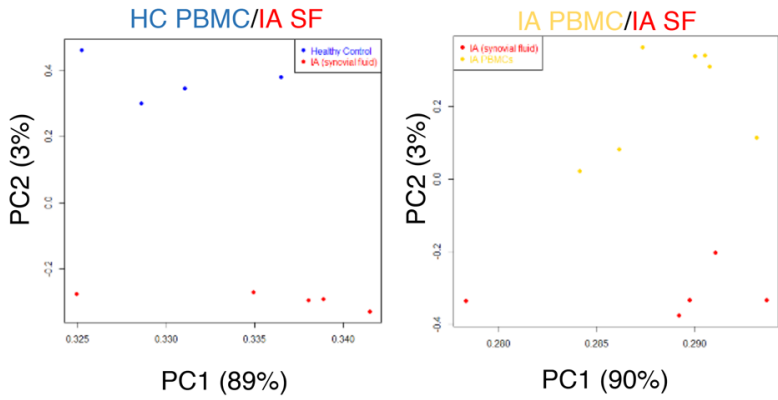

E

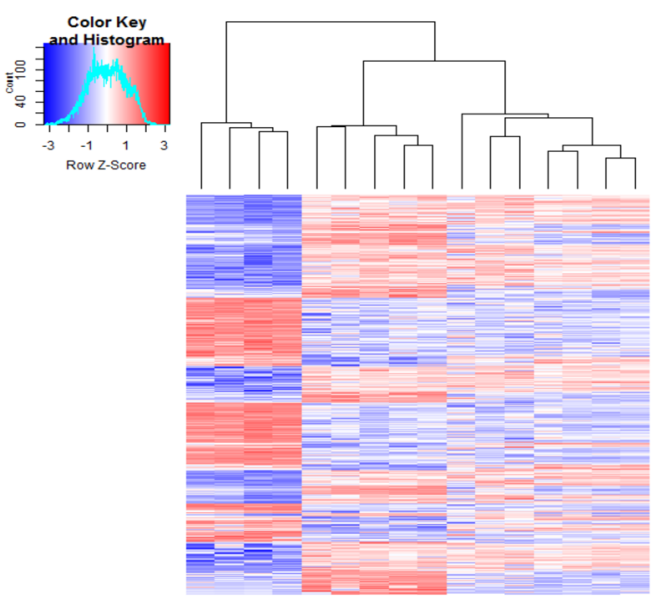

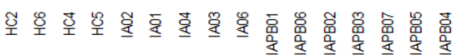

Figure 2. CD141+ DC are enriched in inflamed synovium and are distinct from blood DC. (A) Percentage of CD141+ DC within the DC population of inflammatory arthritis (IA) whole blood (WB) and IA synovial fluid (SF). Square dots represent RA patients; triangles represent PsA patients. (B) Gating strategy

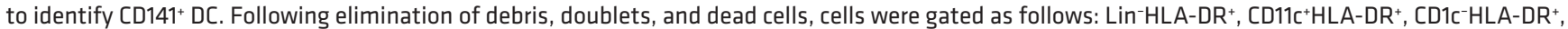
CD141+Clec9A+, and CD141+XCR-1+ cells. (C) RNA from sorted CD141+ DC and CD1C ${ }^{+} \mathrm{HC} / \mathrm{IA} \mathrm{PBMC/SF}$ were sequenced and analyzed for the expression of XCR-1, TLR3, CADM, Clec9A, Clec10A, and CD1c using RNA sequencing. (D) PCA was performed on the total datasets of HC PBMC CD141+ DC, IA SF CD141+ DC, and IA PBMC CD141+ DC. Data shown are from 4 HC donors, 5 IA SF donors, and 7 IA blood donors. (E) Unsupervised hierarchical clustered heatmap of differentially expressed genes in HC peripheral blood CD141+ DC, IA PBMC CD141+ DC, and IA SF CD141 $1^{+}$C. ${ }^{* *} P<0.01$ significantly different from control. WB, whole blood; SF, synovial fluid; IA, inflammatory arthritis.

IA PB $(n=7)$, and $\mathrm{HC} \mathrm{PB}(n=5)$. Cells were magnetically sorted using a CD141+ $\mathrm{mDC}$ microbead kit, and the purity of sorted cells was assessed using a panel of CD141 specific antibodies. Cells were confirmed as $\mathrm{CD} 11 \mathrm{c}^{+} \mathrm{HLA}-\mathrm{DR}{ }^{+} \mathrm{Clec} 9 \mathrm{~A}^{+} \mathrm{XCR}-1^{+}$and negative for CD1c. Fluorescence minus one (FMO) controls were used to determine gating boundaries, and given the difficulty in obtaining positive XCR-1 staining, an isotype control was also used (Supplemental Figure 4). High-quality mRNA was isolated from these CD141 ${ }^{+}$DC, and RNA-seq was performed. Unsupervised hierarchical clustering and principal component analysis (PCA) of the total gene expression dataset and differentially expressed genes (DEG) demonstrated that IA SF CD $141^{+}$DC clustered separately from PB IA and $\mathrm{HC} C D 141^{+} \mathrm{DC}$ (Figure 2, D and E, and 
A

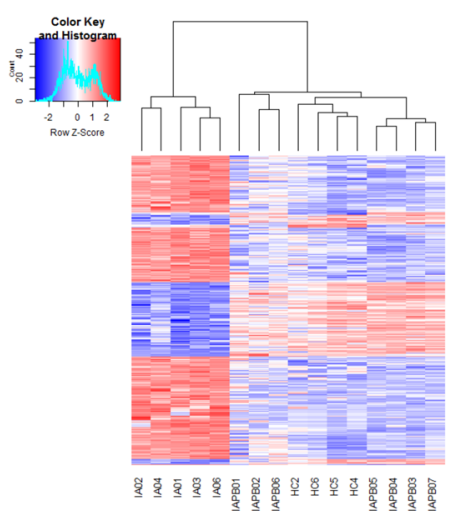

D

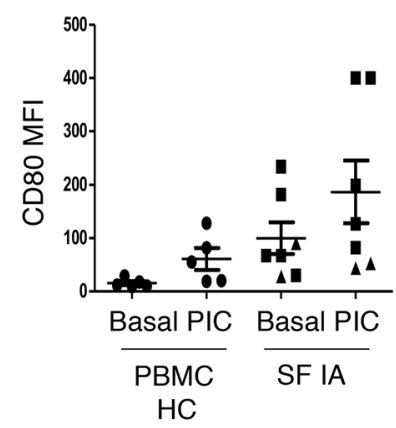

E

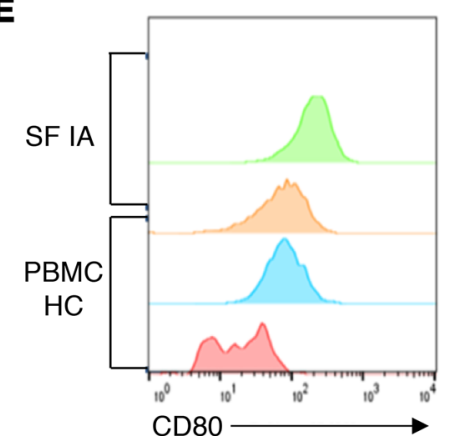

B
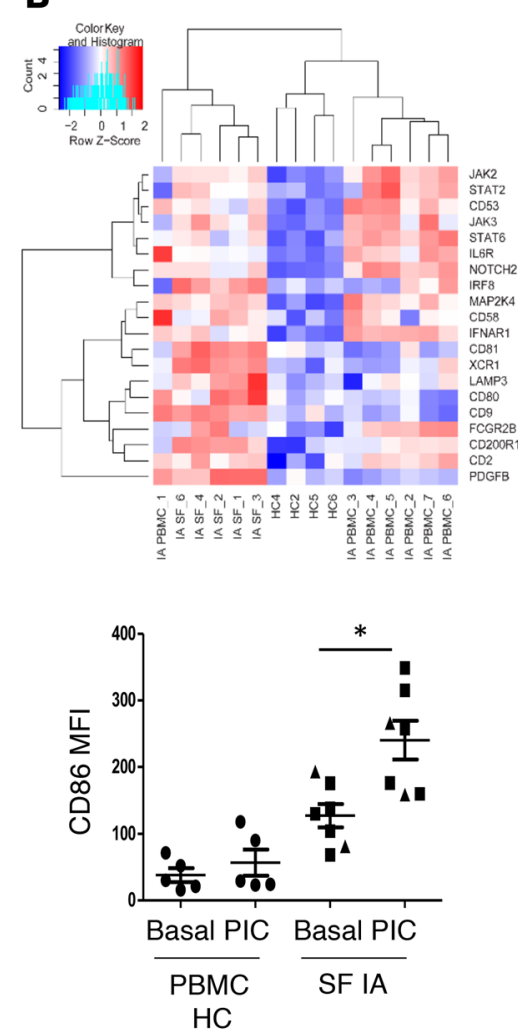

C
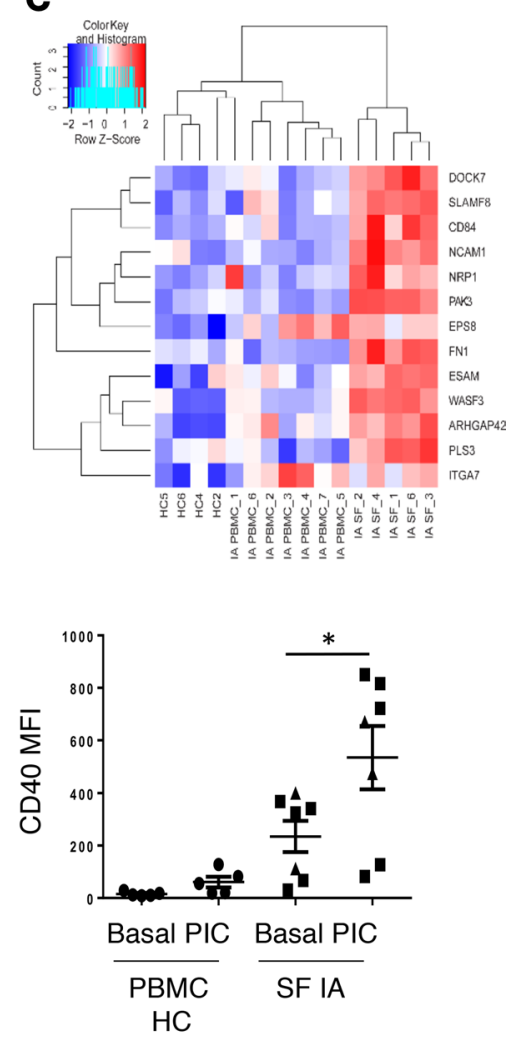
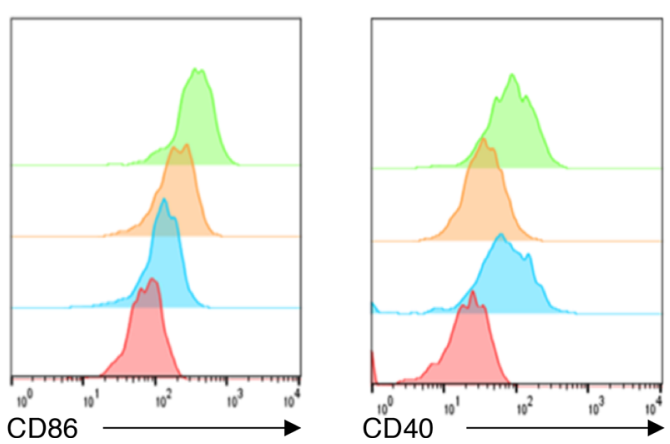

SF IA PIC

SF IA Basal

HC PBMC PIC

HC PBMC Basal

Figure 3. Synovial CD141+ DC have enhanced activation and maturation. Clustered heatmaps displaying (A) unsupervised hierarchical clustering of genes that were distinct in the IA SF CD141+ DC $(n=5)$ compared with either HC $(n=4)$ or IA $(n=7)$ PBMC CD141+ DC. (B and C) Differentially expressed genes (DEGs) involved in immune activation and signaling (B) and cell movement and adhesion (C) in the IA SF CD141+ DC $(n=5)$ compared with either HC $(n=4)$ or IA ( $n=7)$ PBMC CD141 DC. (D) Sorted HC PBMC and SF IA CD141+ DC $(n=5)$ were stimulated overnight with poly (I:C) (PIC) (1 $\mu \mathrm{g} / \mathrm{ml})$, stained for CD80, CD86, and CD40 and analyzed by flow cytometry. Dot plots representing mean fluorescent intensity (MFI) of CD80, CD86, and CD40. Square dots represent rheumatoid arthritis (RA) patients; triangles represent psoriatic arthritis (PsA) patients. (E) Representative histograms of CD80, CD86, and CD40 in HC PBMC and SF IA CD141+ DC. Data were analyzed using the Wilcoxon signed-rank test. ${ }^{*}<0.05$ significantly different from control. SF, synovial fluid; IA, inflammatory arthritis.

Supplemental Figure 5, A and B), indicating the presence of distinct transcriptional variations between all 3 groups. We next performed a pairwise comparison between 3 cell populations - (a) HC PB CD $141^{+}$DC vs. IA PB CD $141^{+} \mathrm{DC}$, (b) HC PB CD $141^{+}$DC vs. IA SF CD $141^{+}$DC, and (c) IA PB CD $141^{+} \mathrm{DC}$ vs. IA SF CD $141^{+}$DC - to identify DEG that were disease and/or site specific. Our whole transcriptome-wide analysis showed that SF IA CD $141^{+}$DC were more closely aligned to HC PB CD $141^{+}$DC compared with IA PB CD $141^{+}$DC (Supplemental Figure 5A). Interestingly, unsupervised hierarchical clustering of DEG demonstrated that all 3 populations cluster separately, showing that $\mathrm{HC} \mathrm{PB} C D 141^{+} \mathrm{DC}$ were transcriptionally distinct from IA PB CD $141^{+}$DC, which were transcriptionally distinct from IA SF CD $141^{+} \mathrm{DC}$, suggesting that there are distinct disease- and site-specific $\mathrm{CD} 141^{+} \mathrm{DC}$ transcripts (Figure 2, D and E). 
Table 1. IPA analysis of enriched pathways in synovial fluid versus peripheral blood CD141+ DC

\begin{tabular}{lcc}
\hline IPA pathways & $-\log (\boldsymbol{P}$ value) & Ratio \\
\hline p53 signaling & 4.87 & 0.31 \\
CD40 signaling & 4.10 & 0.32 \\
\hline RANK signaling in osteoclasts & 0.28 \\
\hline UVA-induced MAPK signaling & 3.40 & 0.28 \\
B cell receptor signaling & 3.39 & 0.24 \\
T cell receptor signaling & 3.32 & 0.27 \\
ERK5 signaling & 3.17 & 0.30 \\
\hline TNFR1 signaling & 3.05 & 0.33 \\
\hline IGF-1 signaling & 3.04 & 0.26 \\
\hline AMPK signaling & 2.96 & 0.22 \\
ERK/MAPK signaling & 2.80 & 0.23 \\
\hline Role of macrophages, fibroblasts and & 2.78 & 0.21 \\
endothelial cells in rheumatoid arthritis & 2.45 & 0.28 \\
\hline Role of JAK1 and JAK3 in $\gamma c$ cytokine signaling & & 0.23 \\
\hline IL-6 signaling & 2.40 & 0.24 \\
\hline PI3K/AKT signaling & 2.27 & 2.25 \\
\hline
\end{tabular}

Ratio column represents ratio of the number of genes from the list that maps to the pathway divided by the total number of genes that map to the same pathway. UVA, Ultra-Violet Radiation.

In Figure 3A, we performed further analysis to examine if there were common DEG distinct in joint CD $141^{+}$DC compared with both the PB HC CD $141^{+}$DC and PB IA CD $141^{+}$DC. This showed that, while there are disease-specific genes, there are also 382 genes in common that are specifically differentially expressed at the site of inflammation compared with HC and IA PB CD $141^{+}$DC.

We further identified 382 genes that were transcriptionally distinct in IASF CD $141^{+} \mathrm{DC}$ compared with both IA PB CD $141^{+}$DC and HC CD $141^{+}$DC, demonstrating a distinct transcriptional signature at the site of inflammation (Figure 3A).

To examine signaling pathways dysregulated in diseased site, Ingenuity Pathway Analysis (IPA; www. qiagen.com/ingenuity) was performed on DEG associated with synovial CD $141^{+} \mathrm{DC}$ and PB CD $141^{+} \mathrm{DC}$. This analysis revealed enrichment in a number of key regulatory and inflammatory pathways that are overrepresented in IA CD $141^{+}$DC compared with PB CD $141^{+}$DC (Table 1). Based on results obtained from the IPA (Table 1), we examined specific genes associated with these pathways, such as cell signaling (JAK2, $J A K 3, S T A T 6)$, immune activation (CD53,CD80), cell adhesion (PLS3,CD84), and cell movement (DOCK7, $W A S F 3$ ), and we noted that these genes were all significantly increased in IA SF CD $141^{+} \mathrm{DC}$ compared with PB CD141 $1^{+}$DC (Figure 3, B and C). This analysis revealed that CD $141^{+}$DC from SF are distinct from IA and HC PB CD $141^{+}$DC. A level of transcriptional diversity between HC and IA PB CD $141^{+}$DC was also observed; however, these were more closely aligned in comparison with IA SFMC CD $141^{+}$DC.

Synovial CD $141^{+}$DC have enhanced activation and maturation. Functional analysis of sorted CD $141^{+} \mathrm{DC}$ from SF and HC PB demonstrated higher expression of DC maturation markers CD80, CD86, and CD40 in synovial CD141 ${ }^{+}$DC. Additionally, we demonstrated increased expression of CD80, CD86, and CD40 and significant induction of CD86 and CD40 expression in response to TLR3 activation by flow cytometry (both $P<0.05$, Figure 3, D and E), suggesting that while SF CD $141^{+} \mathrm{DC}$ reside in a more activated state, they are capable of further maturation in the inflamed joint. We also examined the response of both $\mathrm{HC}$ and IA PB CD $141^{+}$DC to TLR3 stimulation (Supplemental Figure 6). These data revealed that both HC and IA PB respond to TLR3 stimulation to a similar degree, suggesting that there is a degree of similarity between both the $\mathrm{HC}$ and IA PB CD $141^{+} \mathrm{DC}$ response. However, due to the rarity of CD141 $\mathrm{DC}$ in IA patient $\mathrm{PB}$ and the limited amount of blood that is ethically possible to collect from IA donors, further functional downstream analysis directly compared synovial CD $141^{+} \mathrm{DC}$ with HC PB CD $141^{+} \mathrm{DC}$.

Synovial $C D 141^{+} D C$ induce enhanced $C D 4^{+} T$ cell responses. To evaluate the functional effects of synovial CD $141^{+} \mathrm{DC}$ on $\mathrm{T}$ cell responses, an allogeneic coculture assay was performed using sorted synovial CD $141^{+}$DC from joints of IA patients compared with PB CD $141^{+}$DC $(n=4)$. Synovial CD $141^{+}$ DC induced higher levels of granulocyte-macrophage colony-stimulating factor (GMCSF) from CD $4^{+}$ 
T cells $(\mathrm{p}=0.0580)$ compared with $\mathrm{PB} C \mathrm{CD} 141^{+} \mathrm{DC}$ (Figure 4). Moreover, synovial CD141 ${ }^{+} \mathrm{DC}$ induce significantly higher levels of the proinflammatory cytokines IFN- $\gamma$ and TNF- $\alpha$ (Figure $4 ; P<0.01$ and $P<0.05$, respectively). Although not statistically significant, synovial CD $141^{+} \mathrm{DC}$ also induced higher levels of GMCSF, IFN- $\gamma$, and TNF- $\alpha$ from CD $8^{+} \mathrm{T}$ cells compared with PB CD $141^{+} \mathrm{DC}$ (Supplemental Figure 7).

Synovial $C D 141^{+} D C$ induce enhanced $C D 4^{+} T$ cell responses in autologous cocultures. Our data suggest that synovial $\mathrm{CD} 141^{+} \mathrm{DC}$ are involved in actively propagating inflammation in the IA joint. To demonstrate this, we performed autologous $\mathrm{CD} 141^{+} \mathrm{DC}$ cocultures with synovial CD3 ${ }^{+} \mathrm{T}$ cells to recapitulate the $\mathrm{DC}-\mathrm{T}$ cell interactions that occur in the joint $(n=3)$. Synovial $\mathrm{CD}^{+}$and $\mathrm{CD} 8^{+} \mathrm{T}$ cells isolated directly from the joints exhibit some degree of proliferation and proinflammatory cytokines. However, when these synovial $\mathrm{T}$ cells are cocultured with autologous synovial CD $141^{+} \mathrm{DC}$, there is an increase in $\mathrm{T}$ cell proliferation and proinflammatory cytokines from $\mathrm{CD}^{+}$and $\mathrm{CD}^{+} \mathrm{T}$ cells (Figure 5 and Supplemental Figure 8, respectively). Due to the heterogeneity in these patients, the response levels measured display some degree of variation. However, the most reproducible effect between each patient was an increase in $\mathrm{CD}^{+} \mathrm{T}$ cell proliferation and the expression of IFN- $\gamma$ from CD4 ${ }^{+} \mathrm{T}$ cells (Figure 5). However, some patients $(n=2)$ showed variable degrees of increase in TNF- $\alpha$ and IL-17a when synovial T cells were cocultured with synovial CD $141^{+} \mathrm{DC}$ compared with T cells alone.

$C D 141^{+} D C-T$ cell interaction can enhance synovial fibroblast activation. The inflamed synovium consists of a complex interplay between infiltrating leucocytes, resident macrophages, and stromal cells such as synovial fibroblasts. Therefore, we next examined the effects of CD141+ DC and T cell cocultures on synovial fibroblast function - the key invasive cell in the joint. Synovial fibroblasts were cultured with supernatants from the $\mathrm{CD} 141^{+} \mathrm{DC}$ coculture reaction, termed conditioned media, and fibroblast activation was assessed. Conditioned media from $\mathrm{CD} 141^{+} \mathrm{DC}-\mathrm{T}$ cell cocultures significantly increased secretion of IL-6 $(P<0.05)$ and the matrix metalloproteinases MMP-1 $(P<0.01)$ and MMP-3 $(P<$ 0.05 ) in synovial fibroblasts (Figure 6A). Conditioned media from $\mathrm{T}$ cells cultured alone had no significant effects on IL-6 and MMP-1, but they did have an effect on MMP-3 (Supplemental Figure 9A). The levels of IL-6 from T cell conditioned media was quantified, and subsequent levels in K4IM supernatants were corrected to distinguish between T cells and K4IM-derived IL-6 (Supplemental Figure 9B). Furthermore, CD $141^{+}$DC-induced T cell activation resulted in an increase in cell-surface expression of adhesion molecule ICAM on synovial fibroblasts with no change observed for VCAM or the osteoclast differentiation ligand RANKL (Figure 6, B and C, and Supplemental Figure 9). Therefore, these results suggest that $\mathrm{CD} 141^{+} \mathrm{DC}$ cocultures induce secretion of soluble mediators from activated $\mathrm{T}$ cells, which in turn induce invasive phenotypic characteristics in synovial fibroblasts.

Synovial CD141 $1^{+}$DC express the hypoxia marker TREM-1, activation of which further enhances their activation. We next investigated the possible mechanisms underlying enhanced synovial CD $141^{+} \mathrm{DC}$ activation. Previous studies have reported that the inflamed synovial joint is profoundly hypoxic with the mean $\mathrm{pO}_{2}$ level at $3.2 \%$ (12). We therefore hypothesized that TREM-1, a hypoxia-inducible marker known to induce DC maturation, may be involved. Histological analysis demonstrated a significant increase in TREM- $1^{+}$cells in both the SL and LL regions of RA ST versus OA (Figure 7A; $P<0.05$ ). TREM-1 SL expression significantly correlated with in vivo macroscopic synovitis $(r=0.704, P=$ 0.0230). Additionally, a significant increase in CD $141^{+} \mathrm{TREM}-1^{+} \mathrm{DC}$ was demonstrated in IA SF compared with $\mathrm{PB}$ CD $141^{+} \mathrm{DC}$ (Figure 7B; $P<0.05$ ). Immunofluorescence staining for filamentous actin of sorted CD $141^{+}$DC demonstrated typical DC morphology with multiple dendritic protrusions. Upon engagement of the TREM-1 receptor on synovial CD141 ${ }^{+}$DC using an anti-TREM-1 antibody, cytoskeleton structural changes were observed, where TREM-1 stimulated CD $141^{+} \mathrm{DC}$ displayed a more activated phenotype, evident by an increase in veiled projections protruding from the actin cytoskeleton (Figure 7C), indicative of cell movement. TREM-1-activated SF CD $141^{+}$DC have significantly higher cell surface expression of CD80, CD86, and CD40 (Figure 7D; all $P<0.05$ ), whereas HC PB CD $141^{+}$DC, which do not express TREM-1, do not respond to TREM-1 ligation (Supplemental Figure 10). TREM-1 activated DC were subsequently cocultured with allogeneic $C D 3^{+} \mathrm{T}$ cells, where induction of IFN- $\gamma$ and IL-17a was observed (Figure 7E). Furthermore, TREM-1-activated DC also display increased phosphorylation in p38 MAPK (Figure 7F), highlighting downstream signaling pathways that may mediate these specific TREM-1-mediated DC effects. 

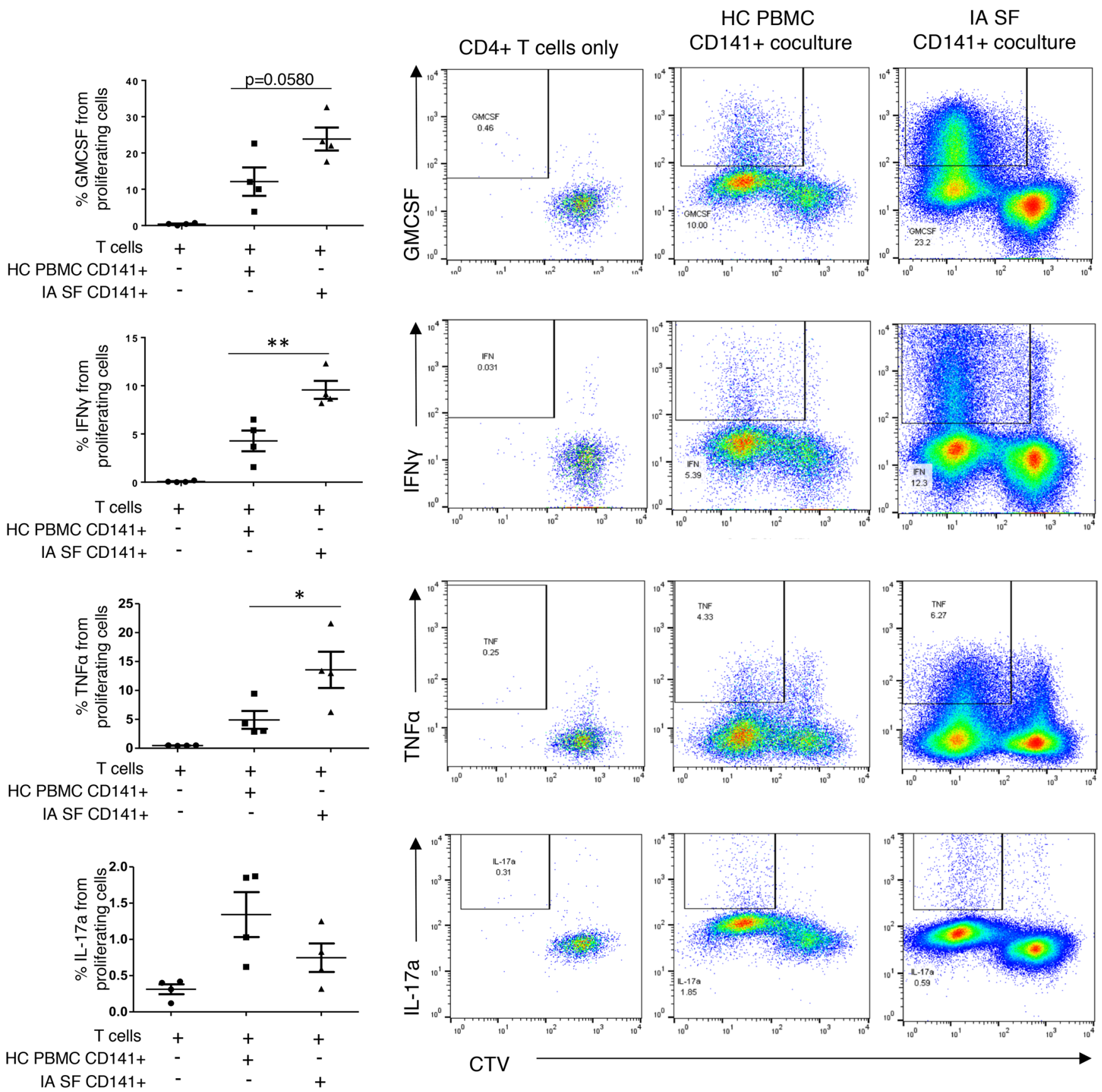

Figure 4. Synovial CD141+ DC induce enhanced CD4 ${ }^{+}$T cell responses. Sorted IA synovial fluid (SF) CD141+ DC $(n=4)$ or HC PBMC CD141+DC were cocultured with allogeneic $C D 3^{+} T$ cells from a single donor for 6 days at a ratio of 1:10, after which proliferation and cytokine production was assessed by flow cytometry. The frequency of proliferating cells was measured by CellTrace Violet (CTV) dilution. After 6 days of coculture, CD ${ }^{+}$T cells were restimulated with PMA/ ionomycin in the presence of brefeldin A; stained intracellularly with fluorochrome-conjugated antibodies specific for GMCSF, IFN- $\gamma$, TNF- $\alpha$, and IL-17a; and analyzed by flow cytometry. $\mathrm{CD} 4^{+}$cells were gated on $\mathrm{CD} 3^{+} \mathrm{CD} 8^{-}$cells. Representative flow cytometry plots showing cytokine staining in $\mathrm{CD} 4^{+} \mathrm{T}$ cells and dot plots representing the percentage of indicated cytokine within the proliferating CD4+ population. Square dots represent rheumatoid arthritis (RA) patients; triangles represent psoriatic arthritis (PsA) patients. Data were analyzed using an unpaired Student's $t$ test. ${ }^{*} P<0.05$, ${ }^{*} P<0.01$ significantly different from control. IA, inflammatory arthritis.

\section{Discussion}

In this study, we examined the phenotypic, functional, and mechanistic role of human CD141 ${ }^{+} \mathrm{DC}$ in autoimmunity. This is the first study to our knowledge that identifies enrichment of CD141+ DC in any subtype of rheumatic diseases at the site of inflammation and specifically highlights their contribution to synovial inflammation. We identified significant enrichment of CD $141^{+} \mathrm{DC}$ in the inflamed synovial joint, which had a distinct transcriptional signature compared with IA and $\mathrm{HC}$ blood $\mathrm{CD} 141^{+}$DC. However, IA PB CD141 ${ }^{+}$ 

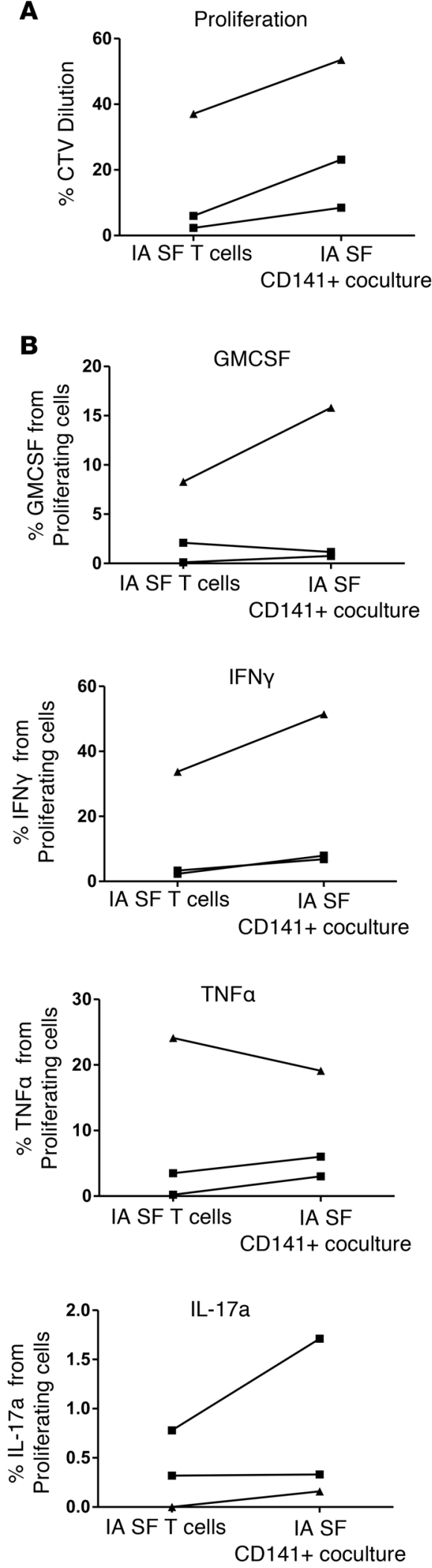

IA SF

T cells alone
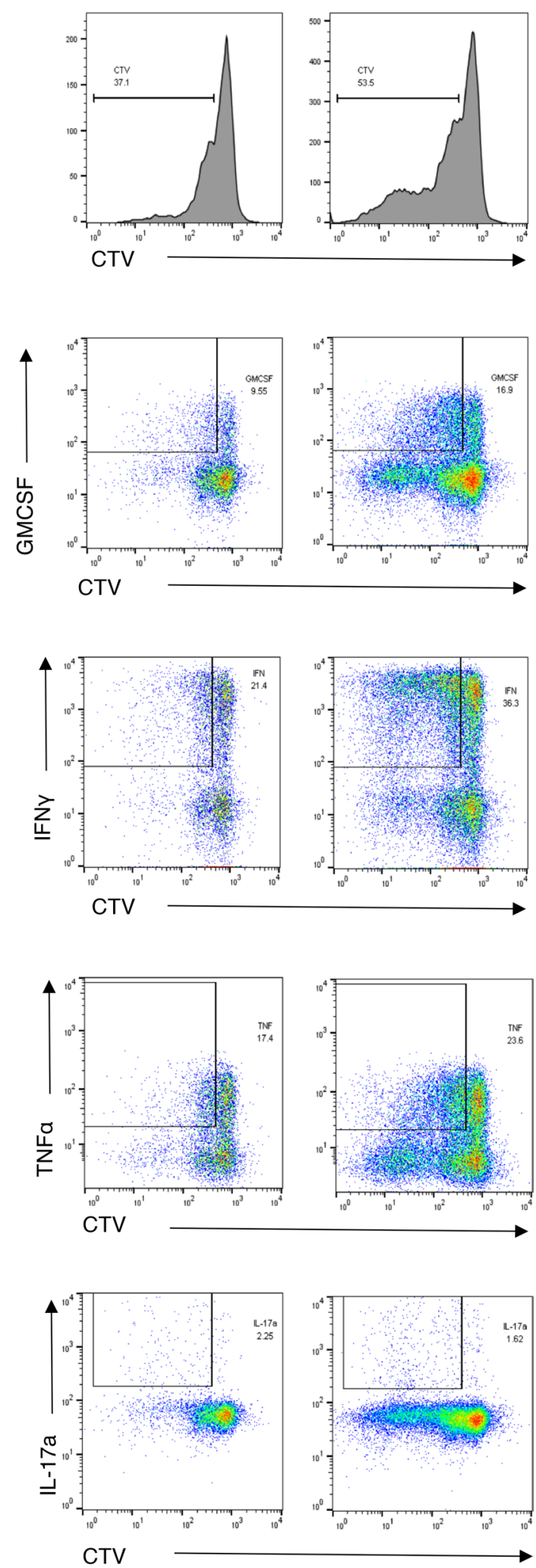

Figure 5. Synovial CD141+ DC induce enhanced $\mathrm{CD4}^{+} \mathrm{T}$ cell responses in autologous cocultures. Inflammatory arthritis (IA) synovial fluid (SF) $\mathrm{CD}^{+} \mathrm{T}$ cells were sorted and cultured alone or with autologous IA SF CD141 ${ }^{+}$ $D C(n=3)$ for 4 days at a ratio of 1:10, after which proliferation and cytokine production was assessed by flow cytometry. The frequency of proliferating cells was measured by CTV dilution. After 4 days of coculture, $\mathrm{CD}^{+} \mathrm{T}$ cells were restimulated with $\mathrm{PMA}$ /ionomycin in the presence of brefeldin A; stained intracellularly with fluorochrome-conjugated antibodies specific for IFN- $\gamma$, TNF- $\alpha$, GMCSF, and IL-17a; and analyzed by flow cytometry. (A) Dot plots representing the percentage of proliferating cells in $\mathrm{CD} 4^{+} \mathrm{T}$ cell populations and representative histograms showing the dilution of CTV. (B) Representative flow cytometry plots showing cytokine staining in $\mathrm{CD} 4^{+} \mathrm{T}$ cells and dot plots representing the percentage of indicated cytokine within the proliferating $C D 4^{+}$population. Square dots represent rheumatoid arthritis (RA) patients; triangles represent psoriatic arthritis (PsA) patients. 
A

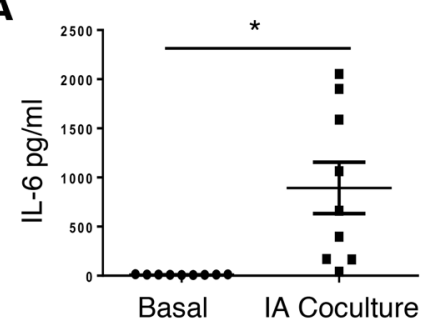

B

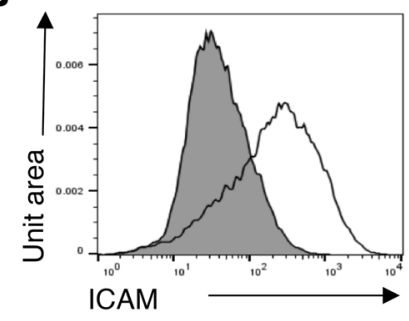

C

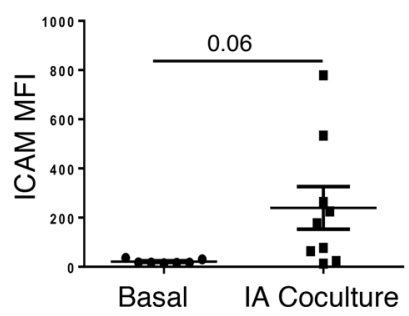

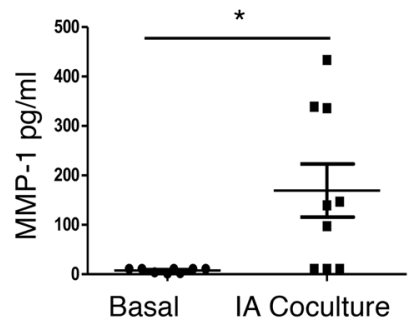
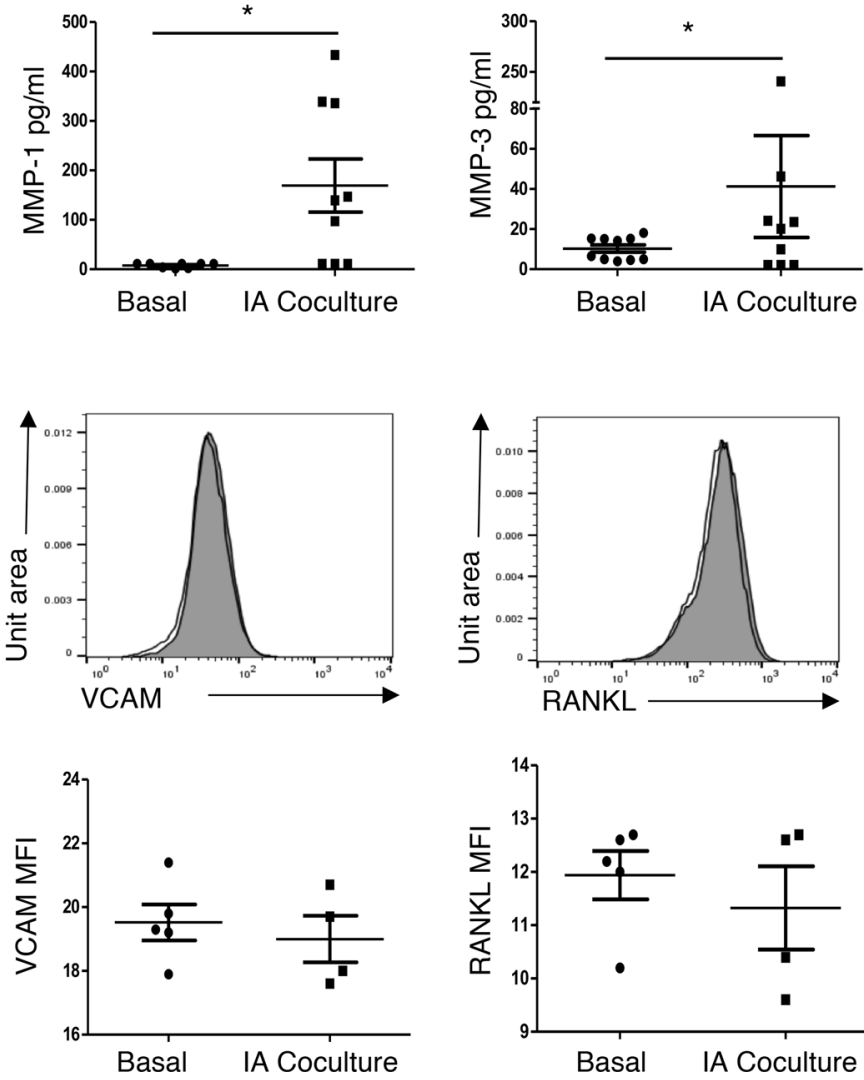

Figure 6. Synovial CD141+ DC induce CD4 ${ }^{+}$and $\mathrm{CD8}^{+} \mathrm{T}$ cell responses, which subsequently activates synovial fibroblasts. K4IM cell line was treated with conditioned media (25\%) from IA SF CD141+ DC and IA SF T cell cocultures $(n=4-9)$ or $25 \%$ normal media for 24 hours. (A) Supernatants were harvested and analyzed for IL-6, MMP-1, and MMP-3 by multiplex ELISA. K4IM were stained with fluorochrome-conjugated antibodies specific for ICAM, VCAM, and RANKL. (B) Representative histograms of ICAM, VCAM, and RANKL staining. (C) Dot plots representing the MFI of ICAM, VCAM, and RANKL on K4IM cells. Data were analyzed using a paired Student's $t$ test. ${ }^{*} P<0.05$ significantly different from control. IA, inflammatory arthritis.

DC also clustered separately from HC blood CD141 ${ }^{+} \mathrm{DC}$, suggesting that there are distinct disease- and site-specific CD $141^{+}$DC transcripts. Further functional analysis demonstrated that, in synovial CD $141^{+} \mathrm{DC}$ there are significantly more activated, as observed by high expression of genes involved in immune activation, cell signaling, cell adhesion, and cell movement. In addition, synovial CD $141^{+} \mathrm{DC}$ exhibited increased responsiveness to TLR3 compared with that of both IA and HC blood CD141 $1^{+} \mathrm{DC}$. We demonstrated that synovial $\mathrm{CD} 141^{+} \mathrm{DC}$ induced higher levels of $\mathrm{CD} 4^{+}$and $\mathrm{CD} 8^{+} \mathrm{T}$ cell activation compared with their $\mathrm{HC} \mathrm{PB}$ counterparts, as made evident by increased expression of IFN- $\gamma$, TNF- $\alpha$, and GMCSF. Autologous synovial $\mathrm{CD} 141^{+} \mathrm{DC}$ cocultures also induce higher levels of synovial $\mathrm{T}$ cell proliferation and cytokine expression, further highlighting the contribution of $\mathrm{CD} 141^{+} \mathrm{DC}$ to synovial inflammation. Furthermore, synovial $\mathrm{CD} 141^{+} \mathrm{DC}-\mathrm{T}$ cell interactions had the ability to further activate synovial fibroblasts, inducing adhesive and invasive pathogenic mechanisms. One potential mechanism identified by which synovial CD141 ${ }^{+} \mathrm{DC}$ mature, and subsequently activate, $\mathrm{T}$ cells is through engagement with TREM-1 via p38.

We report a significant decrease in $\mathrm{mDC}$ in $\mathrm{PB}$ in $\mathrm{IA}$, and specifically, we demonstrate that this overall decrease in $\mathrm{mDC}$ frequency in the periphery may be attributed to a reduction in the $\mathrm{CD} 141^{+} \mathrm{DC}$ population. A reduction in circulating peripheral $\mathrm{mDC}$ is suggestive of increased migration to the synovium or, indeed, lack of release of $\mathrm{mDC}$ progenitors from the BM. Indicative of the former, levels of DC are significantly higher in $\mathrm{ST}$ and fluid compared with $\mathrm{PB}$, thus suggesting migration of circulating $\mathrm{DC}$ to the synovial compartment, where they reside in a more mature state. This is consistent with studies reporting decreased $\mathrm{mDC}$ in IA circulation with a concomitant increase in $\mathrm{SF} \mathrm{mDC}$ with differential maturation phenotypes $(11,13)$. Page et al. identified DC at 3 individual stages of maturation in RA ST (i.e., immature DC, fully mature DC, and an intermediate DC population; ref. 10). Pettit et al. reported that DC, defined as $\mathrm{CD} 33^{+} \mathrm{CD} 14^{-}$cells, expressed low levels of RelB in PB DC compared with high levels in synovial DC, 
A

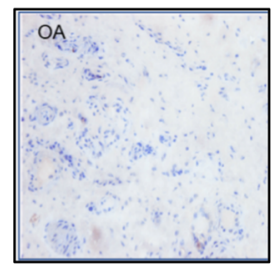

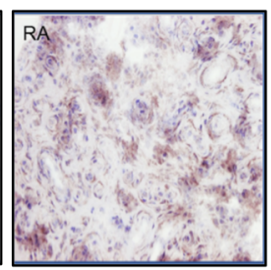

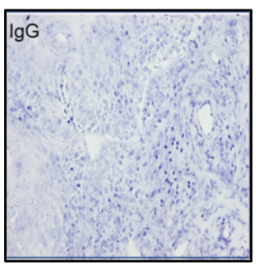

C

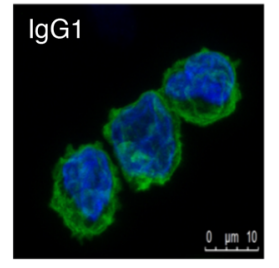

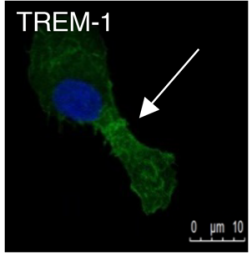
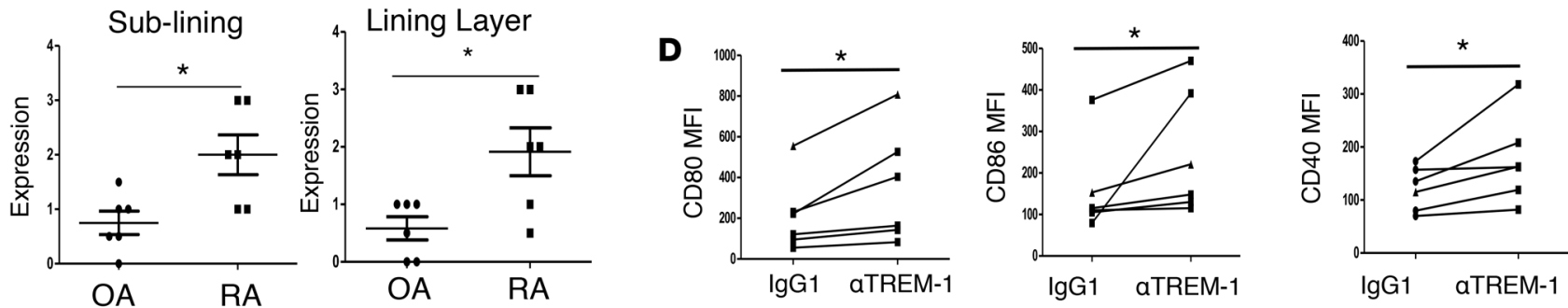

B
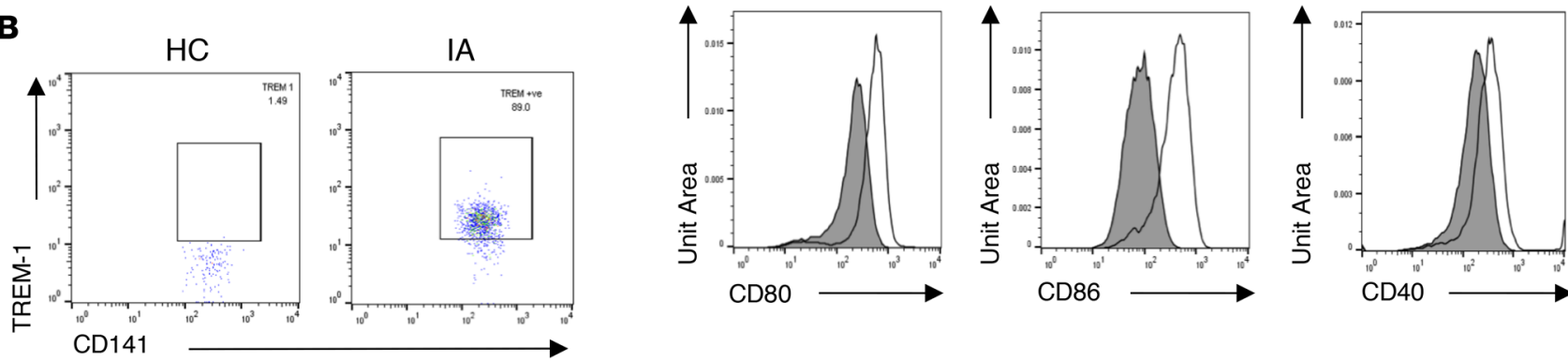

$\mathbf{E}$
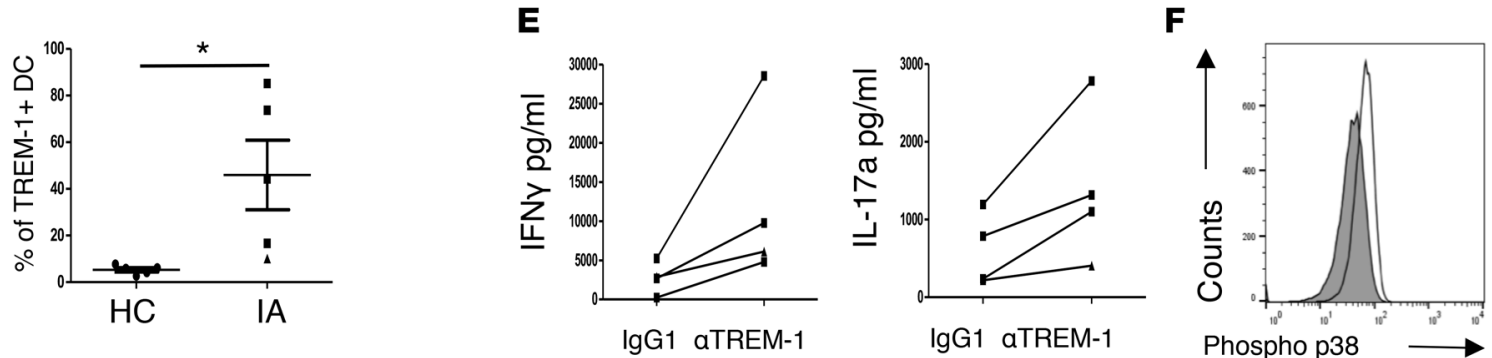

Figure 7. TREM-1+ Synovial CD141+ DC have enhanced activation. (A) Representative images of immunohistochemical staining and semiquantification for TREM- 1 in the sublining layer and the lining layer of rheumatoid arthritis (RA) and osteoarthritis (OA) patients $(n=6)$. Original magnification, 10x. Sorted healthy control (HC) PBMC and inflammatory arthritis (IA) synovial fluid (SF) CD141+ DC were stained with a panel of fluorochrome-conjugated antibodies and analyzed by flow cytometry. CD141+ DC were gated on CD141+HLADR+Clec9A ${ }^{+}$, and the expression of TREM-1 was assessed on these cells. (B) Representative flow cytometry plots showing TREM-1 staining and the percentage of TREM-1+CD141+ DC within HC PBMC and IA SF $(n=5)$. (C) F-actin staining on sorted synovial CD141+ DC stimulated via plate-bound $\alpha$ TREM-1 or IgG1 control for 24 hours. (D) MFI of CD80, CD86, and CD40 on synovial CD141+ DC stimulated via plate-bound $\alpha$ TREM-1 or IgG1 control for 24 hours $(n=6)$. Representative histogram displaying CD80, CD86, and CD40 expression. (E) IA SF CD141+ DC $(n=4)$ stimulated via TREM-1 or IgG1 control for 24 hours were subsequently cocultured with allogeneic CD3 ${ }^{+} T$ cells for 6 days, after which supernatants were harvested and the production of IFN- $\gamma$ and IL-17a were measured by ELISA. (F) Histogram representing increased phosphorylated p38 in TREM-1-activated IA SF CD141+ DC ( $n=1$ ). Phosphorylated p38 (via Tyr182) was detected in IA SF CD141+ DC stimulated via plate-bound $\alpha$ TREM-1 or IgG1 control for 16 hours. Data were analyzed using Mann-Whitney $U$ test. ${ }^{*} P<0.05$ significantly different from control. TREM-1, triggering receptor expressed on myeloid cells 1; IA, inflammatory arthritis.

suggesting that synovial DC have a more activated phenotype (9). Furthermore, transcriptomics identified HLA-DR ${ }^{+} \mathrm{CD} 11 \mathrm{c}^{+} \mathrm{BDCA}-1^{+} \mathrm{DC}$ in RA SF with distinct maturation properties compared with $\mathrm{PB} \mathrm{DC}(14)$ Other studies have also reported that $\mathrm{SF} \mathrm{mDC}$ display a semimature phenotype, with further induction of maturation upon TLR stimulation (11). IPA revealed multiple pathways enriched in synovial CD $141^{+} \mathrm{DC}$, including p53, MAPK, and JAK/STAT signaling pathways, all of which are known to play key roles in driving inflammation in the synovial joint. Previous studies have highlighted these pathways in DC activation/maturation, $\mathrm{T}$ cell differentiation, antigen presentation, and synovial fibroblast invasion (15-17). In conjunction with these studies, the present study demonstrates, for the first time to our knowledge, that $\mathrm{CD} 141^{+} \mathrm{DC}$ are not only enriched in inflamed joints, but also display a more mature phenotype to PB DC. 
PCA and unsupervised hierarchical clustering analysis revealed that CD $141^{+} \mathrm{DC}$ are transcriptionally distinct in IA SF compared with those in the circulation (IA PB or HC PB). RNA-seq analysis identified multiple DEG between blood (HC and IA) and synovial CD $141^{+}$DC involved in DC maturation and function. Specifically, we identified significant increases in genes known to be involved in antigen presentation $(18,19)$, maturation (20-23), migration via Rho GTPase signaling (19), immunological synapse formation $(24,25)$, and adhesion $(26,27)$. Hierarchical clustering analysis of DEG demonstrated that all 3 populations cluster separately, suggesting that there are distinct disease- and site-specific CD $141^{+} \mathrm{DC}$ transcripts.

Previous studies have highlighted the superior ability of $\mathrm{CD} 141^{+} \mathrm{DC}$ to cross-present soluble antigen to $\mathrm{CD}^{+} \mathrm{T}$ cells (1-3). While outside the constraints of this study, we demonstrated that CD141 ${ }^{+} \mathrm{DC}$ from SF induced higher levels of IFN- $\gamma$, TNF- $\alpha$, and GMCSF compared with HC PB CD $141^{+}$DC, all of which have important destructive implications for joint inflammation. In addition to this, autologous CD $141^{+} \mathrm{DC}$ cocultures highlight the proinflammatory effect that these synovial DC have on synovial $\mathrm{T}$ cell response. While CD86 and CD80 expression were similar under basal conditions and in response to poly (I:C) in HC $\mathrm{PB} \mathrm{CD} 141^{+} \mathrm{DC}$ and IA PB CD $141^{+} \mathrm{DC}$, the CD40 surface expression was higher in IA PB CD $141^{+} \mathrm{DC}$ compared with HC PB CD $141^{+}$DC. This suggests that - while both HC and IA CD $141^{+} \mathrm{DC}$ are in a semimature state, consistent with previous reports on circulating DC in RA (28) - the increase in CD40 expression indicates that IA PB CD $141^{+} \mathrm{DC}$ may exist in a primed state. Therefore, distinct functional differences are likely to exist between $\mathrm{HC}$ and IA PB CD $141^{+}$DC. However, due to the rarity of these cells and ethical restraints on the amount of blood that can be taken from patients, a direct comparison of IA PB CD $141^{+} \mathrm{DC}$ and IA SF CD $141^{+}$DC on T cell activation was not feasible and is recognized as a limitation of the study.

Our data describe a role for $\mathrm{CD} 141^{+} \mathrm{DC}$ in the joint, identifies these cells in the synovium, and highlights their pathogenic and proinflammatory capacity. Other DC may also contribute to driving synovial inflammation, including $\mathrm{CD} 1 \mathrm{c}^{+} \mathrm{DC}$; however, few studies have examined their pathogenic role in the context of the inflamed joint. Only 1 previous study has identified CD1c-like proinflammatory DC in the joint where it was demonstrated that they can also contribute to T cell-mediated inflammation (14). While outside the scope of this study, future studies comparing the pathogenic role of $\mathrm{CD} 1 \mathrm{c}^{+}$and $\mathrm{CD} 141^{+} \mathrm{DC}$ will give more insight to their relative contribution to disease. While the efficacy of TNF-targeting therapy has been well established, Mavrilimumab - a GMCSF receptor $\mathrm{mAb}$ - has been shown to reduce disease activity in RA (29). The ability of $\mathrm{CD} 141^{+} \mathrm{DC}$ to induce both $\mathrm{CD} 4^{+}$and $\mathrm{CD} 8^{+} \mathrm{T}$ cell proliferation has implications for IA that are associated with aberrant $\mathrm{T}$ cell responses (30-32). Genetic susceptibility to RA is associated with HLA class II genes in the MHC complex, and the presence of multiple CD4 ${ }^{+}$Th subsets in SF and tissue have been identified (33-35). Recent studies have identified T cells within the RA synovium that express high levels of PD-1 and promote B cell responses (36). Furthermore, synovial CD161 ${ }^{+}$ Th17 lineage cells that are resistant to Treg-mediated suppression have also been identified in RA (37).

Few studies exist on CD141 ${ }^{+} \mathrm{DC}$ in disease. This is due to the small numbers of CD $141^{+} \mathrm{DC}$ in blood and the unique challenge of isolating these cells from tissues. However, the present study is the first to our knowledge to report their enrichment and function in the inflamed joint. Seminal studies published in 2010, dissecting the function of $\mathrm{CD} 141^{+} \mathrm{DC}$ and their homology to mouse CD8 ${ }^{+} \mathrm{DC}$, greatly improved our understanding of $\mathrm{CD} 141^{+} \mathrm{DC}$ biology. $\mathrm{PB} \mathrm{CD} 141^{+} \mathrm{DC}$ are superior at cross-presentation of antigen to $\mathrm{CD}^{+} \mathrm{T}$ cells, have the capacity to induce $\mathrm{CD} 4^{+}$and $\mathrm{CD} 8^{+} \mathrm{T}$ cell proliferation, express high levels of TLR3, and produce IL-6, TNF- $\alpha$, IL-12p70, IFN- $\gamma$, and CXCL10 (1-3). In the context of disease, CD141 ${ }^{+}$ DC have been investigated predominantly in the field of cancer biology due to their ability to cross-present antigen to $\mathrm{CD}^{+} \mathrm{T}$ cells. Specifically, Clec9A antibodies that deliver tumor antigen to $\mathrm{CD} 141^{+} \mathrm{DC}$ have been developed to induce tumor-specific $\mathrm{CD}^{+}$cytotoxic $\mathrm{T}$ cell responses (38), and high expression of $\mathrm{PD}-\mathrm{L} 1^{+} \mathrm{CD} 141^{+} \mathrm{DC}$ was previously reported in patients with multiple myeloma (39). The potential role of $\mathrm{CD} 141^{+} \mathrm{DC}$ in cancer biology is understandable, given their effects on cytotoxic $\mathrm{T}$ cell responses. However, how this population contributes to autoimmunity is less clear. It is now understood that Clec9A can recognize dead cells, via F-actin, and facilitates cross-presentation to $\mathrm{CD} 8^{+} \mathrm{T}$ cells (40). Given their unique role in phagocytosing dead or necrotic cell antigens, we hypothesize that CD $141^{+} \mathrm{DC}$ are recruited to the joint in response to ongoing cell death and damage within the synovium. Indeed, the chemokine XCL-1, known to recruit $\mathrm{CD} 141^{+} \mathrm{DC}$, has previously been identified in the synovium (41).

Our data also highlight the complex interplay between the pathogenic stromal cells of the synovium, the synovial fibroblast, and infiltrating leucocytes. Conditioned media from CD $141^{+} \mathrm{DC}-\mathrm{T}$ cell cocultures led to activation of synovial fibroblasts with increased expression of IL-6, MMP-1, MMP-3, and ICAM, all 
of which further potentiate synovial inflammation and subsequent cartilage degradation. This has important implications for synovial inflammation, as synovial fibroblasts manifest an abnormal phenotype characterized by resistance to apoptosis, with increased proliferation and invasion of adjacent cartilage. This effect is mediated through increased expression of adhesion molecules, MMPs, and over-activity of key signaling pathways (42-44). Furthermore, synovial fibroblasts can spread disease to previously unaffected joints, further highlighting their primed pathogenic phenotype (43). While circulating CD $141^{+} \mathrm{DC}$ may also have the ability to activate synovial fibroblasts, such a scenario would not occur in vivo in the context of RA. Therefore, within the complex ST microenvironment, these data demonstrate a pathologically relevant interaction of the synovial $\mathrm{CD} 141^{+} \mathrm{DC}$ that are enriched at the site of inflammation with $\mathrm{T}$ cells on synovial fibroblast function.

The inflamed joint is profoundly hypoxic (12), and previous studies have shown that in vivo $\mathrm{pO}_{2}$ levels within the joint are inversely associated with synovial infiltrates (45) and immune cell activation (46); therefore, we hypothesized that the unique functional properties of synovial CD $141^{+} \mathrm{DC}$ may, in part, be attributed to the hypoxic joint microenvironment. Furthermore, Bosco et al. identified TREM-1 as a hypoxia-inducible gene on monocyte-derived DC (47). Intriguingly, in this study, we demonstrated the presence of TREM-1 on synovial CD $141^{+}$DC and not blood CD $141^{+}$DC. Activation of TREM-1 on CD141 ${ }^{+}$DC via a cross-linking antibody led to cytoskeleton rearrangements with the appearance of veils, known to be required for immunological synapse formation and $\mathrm{T}$ cell activation $(25,48)$. Subsequently, TREM-1 induced CD $141^{+}$DC maturation with a concomitant increase in IFN- $\gamma$ and IL-17a from T cells. Furthermore, we propose a role for p38 in the induction of these TREM-1-mediated effects. These results are consistent with Bosco et al., who identified TREM- $1^{+} \mathrm{CD} 1 \mathrm{c}^{+}$cells in SF from juvenile idiopathic arthritis (JIA) patients (47), which expressed high levels of HIF1 $\alpha$, further suggesting that immune amplification of DC via TREM-1 is, in part, dependent on hypoxia. The natural ligand for TREM-1 has yet to be identified, mainly due to the rapid dissociation of the receptor and its ligand in vivo. However, proteins such as high mobility group box 1 (HMGB1) released by necrotic cells have been suggested to function as a natural ligand in vivo. Numerous papers have identified HMGB1 in the joint due to the high level of ongoing damage that occurs at this site (49). Therefore, it is conceivable that, via TREM-1 (and Clec9A, which also bind dead cell antigens), CD $141^{+}$DC are activated by DAMPs in the joint.

In conclusion, this study has demonstrated that $\mathrm{CD} 141^{+} \mathrm{DC}$ are enriched in the IA synovium in a more mature state and are transcriptionally distinct from PB CD $141^{+}$DC. Synovial CD $141^{+}$DC have enhanced expression of a number of proinflammatory pathways compared with their $\mathrm{PB}$ counterparts. This mature state may contribute to their ability to induce more proinflammatory $\mathrm{T}$ cells responses, which has downstream consequences for synovial fibroblast invasive mechanisms. We hypothesize that the hypoxic nature of the joint contributes to DC maturation via TREM-1, and CD $141^{+} \mathrm{DC}$ are recruited to the joint in response to damage.

\section{Methods}

Patient recruitment, arthroscopies, and sample collection. Seventy-two patients with active IA were recruited from outpatient clinics at the Department of Rheumatology, St. Vincent's University Hospital and Tallaght Hospital (Dublin, Ireland). The patient demographics for this cohort are included in Table 2. Arthroscopy of the inflamed knee was performed under local anesthetic, using a 2.7-mm needle arthroscope (Richard Wolf Medical Instruments), and macroscopic synovitis was scored. Macroscopic synovitis was assessed under direct visualization at video arthroscopy by the clinician performing the surgical procedure. Macroscopic synovitis was scored using a well-established and validated visual analogue scale of $1-100 \mathrm{~mm}$, as described previously (50). Biopsies were OCT embedded (TissueTek) for immunohistochemical analysis or utilized to establish whole tissue synovial cell suspensions. PB and SFMC were also obtained at arthroscopy or rheumatology clinics (St. Vincent's University Hospital and Tallaght Hospital, Dublin, Ireland).

Cell isolation. ST biopsies were obtained at the time of arthroscopy and mechanically and enzymatically digested using the GentleMacs system (Miltenyi Biotec) using a soft tumor dissociation kit (Miltenyi Biotec), according to the manufacturer's instructions. Samples were stained with a panel of fluorochrome-conjugated antibodies for flow cytometry as described below. In addition, PBMC and SFMC were isolated by density gradient centrifugation (Lymphoprep, Stemcell Technologies) according to the manufacturer's recommendations. $\mathrm{CD} 141^{+} \mathrm{DC}$ were isolated from SFMC using a 2-tiered magnetic sort. CD3 antibody-conjugated magnetic beads (Miltenyi Biotec) were used to deplete CD3 from SFMC. Following this, CD141 magnetic 
Table 2. Patient demographics

\begin{tabular}{lc}
\hline Parameters & Patients $(n=\mathbf{7 2})$ \\
\hline Age (mean \pm SD) & $50( \pm 13)$ \\
DAS28 (mean \pm SD) & $4.2( \pm 1.6)$ \\
Sex: Female & $57 \%$ \\
\hline Male & $43 \%$ \\
Diagnosis: Rheumatoid arthritis & $76 \%$ \\
\hline Psoriatic arthritis & $24 \%$ \\
\hline Treatment: No medication & $39 \%$ \\
\hline Methotrexate alone & $19 \%$ \\
\hline Methotrexate in combination & $18 \%$ \\
\hline Anti-TNF & $25 \%$ \\
\hline Abatacept & $1.4 \%$ \\
\hline Tocilizumab & $1.4 \%$ \\
\hline Rituximab & $1.4 \%$ \\
\hline Steroids & $2.7 \%$ \\
\hline Other & $11 \%$ \\
\hline Synovitis $(\boldsymbol{n}=\mathbf{5 4})$ (mean \pm SD)78.3 \pm 21$)$ & \\
\hline
\end{tabular}

beads (Miltenyi Biotec) were used to purify CD $141^{+} \mathrm{DC}$ from SFMC. Sorted CD $141^{+} \mathrm{DC}$ were examined by flow cytometry and were defined as $\mathrm{Lin}^{-}, \mathrm{HLA}-\mathrm{DR}^{+}, \mathrm{CD} 11 \mathrm{c}^{+}, \mathrm{CD} 1 \mathrm{c}^{-}, \mathrm{CD} 141^{+}, \mathrm{Clec} 9 \mathrm{~A}^{+}$, and XCR-1 ${ }^{+}$

Flow cytometry. DC were identified in ST, SFMC, PBMC, and whole blood using the following flow cytometry staining and gating strategy. Cells were gated based on forward and side scatter, and dead cells and doublets were removed. FMO controls were used to determine gating boundaries. Live Dead Red or Live Dead Aqua (Molecular Probes) was used to eliminate dead cells. To eliminate nonspecific binding of mouse monoclonal antibodies to $\mathrm{Fc}-\gamma$ receptor $(\mathrm{Fc} \gamma \mathrm{R})$ on $\mathrm{DC}$, samples were blocked with a human $\mathrm{F} c \gamma \mathrm{R}$-binding inhibitor prior to antibody staining (eBioscience). The following antibodies were used in a combination of panels to detect DC subsets and activation. Lineage 1 FITC (CD3, clone SK7; CD16, clone 3G8; CD19, clone SJ25C1; CD20, clone L27; CD14, clone M $\varphi$ P9; CD56, clone NCAM16.2; BD catalog 340546) HLA-DR V450 (clone G46-6, BD Biosciences, catalog 561359) CD11c APC-Cy7 (clone BU15, BioLegend, catalog 337217), CD45 FITC (clone HI30, BD Biosciences, catalog 555482), CD45 PERCP-Cy5.5 (clone H130, BD Biosciences, catalog 564105), CD141 BV510 (clone 1A4, BD Biosciences, catalog 563298), CD123 PERCP-Cy5.5 (clone 7G3, BD Biosciences, catalog 560904), CD40 PECy7 (clone 5C3, BD Biosciences, catalog 561215), CD80 PE (clone L307.4, BD Biosciences, catalog 557227), TREM-1 Alexa647 (clone 193015, BD Biosciences, catalog 564472), Clec9A (DNGR-1) PE (clone 20/05-3A4, BD Biosciences, catalog 563488), XCR-1 PE (clone 1097A, R\&D Systems, catalog FAB8571P), Isotype control (R\&D Systems, catalog IC105P), and CD86 FITC (clone 2331, BD Biosciences, catalog 555657).

For DC activation experiments, cells were stimulated with $1 \mu \mathrm{g} / \mathrm{ml}$ poly (I:C) (Invitrogen) for 24 hours, after which the expression of costimulatory markers CD80, CD86, and CD40 was analyzed by flow cytometry. BD Cytofix/Cytoperm kit was used to stain whole blood as per manufacturer's instructions. For the detection of phospho p38, the BD PhosFlow kit with Perm Buffer III was used according to manufacturer's instructions. Permeabilized cells were stained with an antibody specific for phosphorylated p38 (Tyr182) conjugated to APC. In order to adjust for spectral overlap between detectors, compensation was applied using single-stained compensation beads (BD Biosciences). Samples were acquired using the CyAn Flow Cytometer (Beckman Coulter) and analyzed using Flowjo software (Treestar Inc.).

$R N A$-seq. Total RNA was extracted from $\mathrm{CD} 141^{+} \mathrm{DC}$ IA synovial samples, IA $\mathrm{PB}$, and $\mathrm{HC} \mathrm{PB}$, and the quality of all RNA samples was evaluated using an Agilent Bioanalyzer. RNA samples were reverse transcribed, and sequencing libraries were constructed using NuGen Ovation Universal RNA-Seq System (catalog 0402A01) according to the manufacturer's protocol. The resulting sequencing libraries were analyzed using the Caliper LabChip GX and quantified using KAPA qPCR. Libraries were then normalized and pooled in 1 batch of 6 and 1 batch of 5 . Each pool was clustered and sequenced on an Illumina NextSeq500 instrument using $2 \times 100$ bp paired-end reads, following the manufacturer's protocols. The average number of reads per sample was 117 million reads with a minimum of 90.1 million reads. Raw read quality was evaluated using FastQC. 
Reads were trimmed for adaptors and sequence quality. Trimmed reads were aligned to human b37.3 reference genome using Omicsoft sequence aligner. Aligned reads were quantified using EM algorithm with RefSeq transcriptome model accessed on June 6, 2017 (51). Aligned data were evaluated for quality using several quality metrics (e.g., mapping rate, coverage) and visually inspected for samples deviating from the population across multiple metrics and PCA. One of each HC and IA synovial samples were excluded from analysis as signal outliers. Data is available on NCBI GEO following with the accession number GSE108174.

Statistical analysis of RNA-seq data was performed in R version 3.3.1 with the limma package version 3.28.14 (52). Limma Voom package (PMID, 24485249) (53) was used to normalize and identify DEG from counts data. Transcripts with zero counts in more than two-thirds of the samples were discarded from downstream analysis to reduce noise in the expression data. Gene counts were converted to $\log _{2}$ counts per million (cpm), quantile normalized, and precision weighted. RNA-seq gene features were considered differentially expressed if they satisfied a 2 -fold change and FDR $<0.05$ cutoff. FDR control was performed with the Benjamini-Hochberg procedure. Heatmaps were generated in $\mathrm{R}$ with the heatmap. 2 function in the gplots package version 3.0.1. Hierarchical clustering was performed with Ward's linkage. We also noted the presence of 2 outliers within the dataset, $\mathrm{HC} 1$ and IA5. We noted that patient IA5, in addition to IA, had a clinical diagnosis of gout; therefore, we excluded this patient from further downstream analysis.

IPA was used to identify canonical pathways overrepresented within DEG.

IHC analysis and scoring. Synovial sections (7- $\mu \mathrm{m}$ sections) were cut using a cryostat and placed on glass slides coated with 2\% 3-amino-propyl-triethoxy-silane (MilliporeSigma). A routine 3-stage immunoperoxidase labeling technique incorporating the avidin-biotin-immunoperoxidase complex using IHC analysis was performed using the Dako ChemMate Envision Kit. Nonspecific binding was blocked using 1\% casein in PBS for 20 minutes. Rabbit monoclonal CD141 (Abcam; catalog 109189; clone EPR4051), mouse polyclonal Clec9A (Abcam; catalog 104910, clone 8F9) and rabbit monoclonal TREM-1 (Abcam; catalog ab93717, its polyclonal,) primary antibodies were incubated on sections for 2 hours at room temperature. An IgG1 control antibody (Cell Signaling Technologies, catalog 7074) was used as a negative control. Following primary antibody incubation, endogenous peroxidase activity was blocked using $0.3 \% \mathrm{H}_{2} \mathrm{O}_{2}$. Slides were incubated for 1 hour with horseradish peroxidase-conjugated secondary antibody (Dako). Color was developed in diaminobenzidine solution (1:50; Dako) and counterstained with hematoxylin. Slides were mounted in Pertex media and analyzed using an established and validated semiquantitative scoring method (54). Percentage positivity was graded on a $0-4$ scale, where $0=$ no stained cells, $1=1 \%-25 \%$ stained cells, $2=25 \%-50 \%$ stained cells, $3=50 \%-75 \%$ stained cells, and $4=75 \%-100 \%$ stained cells. Section was scored separately for the sublining regions (increased blood vessels and influx of inflammatory/immune), which then migrated toward the invasive outside layer of the synovium (LL), where they stimulated resident fibroblasts and macrophages. For immunofluorescence, cryostat sections were defrosted at room temperature for 20 minutes, fixed in acetone for 10 minutes, and washed in PBS for 5 minutes. Nonspecific binding was blocked using 1\% casein in PBS for 20 minutes. Immunofluorescent staining was performed for rabbit monoclonal antibody CD141 (1:100, Abcam), mouse monoclonal antibody Clec9A (1:50, Abcam, catalog 104910, clone 8F9). Following overnight incubation in a humidified chamber at $4^{\circ} \mathrm{C}$, immunofluorescence sections were coincubated with Alexa Fluor-488-conjugated goat anti-mouse superclonal secondary antibody (1:200, Invitrogen) and Cy3-conjugated goat anti-rabbit secondary antibody (1:500, Jackson ImmunoResearch) for 60 minutes and counterstained with DAPI nuclear stain (1:1,000, MilliporeSigma, catalog 111-165-144) for 10 minutes. Sections were mounted with Antifade (Molecular Probes) and assessed by immunofluorescence microscopy (Olympus BX51; magnification, 20×).

Allogeneic and autologous DC-T cell cocultures. CD141 ${ }^{+} \mathrm{DC}$ were isolated from SF as described above. Allogeneic $\mathrm{CD}^{+} \mathrm{T}$ cells were isolated from healthy donor blood using CD3 microbeads (Miltenyi Biotec) as per manufacturer's instructions or for autologous cocultures, synovial $\mathrm{CD}^{+} \mathrm{T}$ cells were isolated. $\mathrm{T}$ cells were labeled with $5 \mu \mathrm{M}$ Cell Trace Violet (Invitrogen) and cocultured with DC for 6 days for allogeneic cocultures or 4 days for autologous cocultures, after which time, proliferation was measured. T cells and CD $141^{+} \mathrm{DC}$ were cocultured at a ratio of 10:1; at the end of the coculture, cells were then restimulated with $50 \mathrm{ng} / \mathrm{ml} \mathrm{PMA}$ and $500 \mathrm{ng} / \mathrm{ml}$ ionomycin in the presence of Brefeldin A $\left(5 \mu \mathrm{g} / \mathrm{ml}\right.$; all from MilliporeSigma) for 5 hours at $37^{\circ} \mathrm{C}$. Cells were stained extracellularly for CD8 (clone RPA-T8, BD Biosciences, catalog 557746) and CD3 (clone UCHT1, BD Biosciences, catalog 563109) in addition to Live/Dead Red viability dye (Invitrogen) according to manufacturer's instructions. Intracellular cytokines IL-17a (clone BL168, BioLegend, catalog 512307), IFN- $\gamma$ 
(clone B27, BD Biosciences, catalog 562017), GMCSF (clone BVD2-21C11, BD Biosciences, catalog 554507), and TNF- $\alpha$ (clone MAb11, eBioscience, catalog 45-7349-42) were subsequently stained using the Fix/Perm kit (Invitrogen) according to manufacturer's instructions. Samples were acquired using the CyAn Flow Cytometer (Beckman Coulter) and analyzed using Flowjo software (Treestar Inc.).

Synovial fibroblast functional assays. An immortalized normal human synovial fibroblast cell line (K4IM) was utilized to examine the effect of $\mathrm{DC} / \mathrm{T}$ cell coculture conditioned supernatants on fibroblast activation and invasive mechanisms. Cells were rendered quiescent by culturing in serum free media for 10 hours before stimulating with $\mathrm{CD} 141^{+} \mathrm{DC} / \mathrm{T}$ cell coculture supernatants (1:4) for 24 hours. Synovial fibroblasts were stained with fluorochrome-conjugated antibodies specific for ICAM-1 BV421 (clone HA58, BD Biosciences, catalog 564077), VCAM PE (clone 51-10C9, BD Biosciences, catalog 555647), and RANKL APC (clone MIH24, eBioscience, catalog 347507) and analyzed by flow cytometry. Protein concentration of IL-6, MMP1 , and MMP-3 were measured in cultured supernatants by multiplex assay according to the manufacturer's instructions on a custom assay kit (Meso Scale Discovery). Electrochemiluminescence was measured using the Meso Scale Discovery Sector Imager 2400. The levels of cytokine present in coculture conditioned media were measured using this method and subtracted from the overall total cytokine production from K4IM cells.

Immunofluorescent staining for F-actin in $C D 141^{+} D C$. CD $141^{+} \mathrm{DC}$ were isolated from SF as described above. To examine the effect of TREM-1 on CD $141^{+}$DC cytoskeletal assembly/morphology, slides were precoated with $10 \mu \mathrm{g} / \mathrm{ml}$ of anti-TREM-1 mAb (clone 193015, R\&D Systems) or an isotype-matched control antibody (mouse IgG1, R\&D Systems, catalog IC105P) for 2 hours at $37^{\circ} \mathrm{C}$. Cells were plated on precoated slides for 24 hours. Cells were subsequently stained for F-actin. Briefly, chamber slides were rinsed in PBS and fixed in $4 \%$ paraformaldehyde before being permeabilized with $0.1 \%$ Triton X-100. To visualize F-actin fibres, slides were stained at room temperature with Oregon Green 488 Phalloidin (Molecular Probes). Nuclei were counterstained with DAPI (MilliporeSigma); stained cells were visualized with a Leitz DM40 microscope, and images were captured using an AxioCam system with AxioVision 3.0.6 software (Carl Zeiss).

TREM-1 ligation assays. Synovial or $\mathrm{HC} \mathrm{CD} 141^{+} \mathrm{DC}$ were sorted as described above and stimulated via plate-bound anti-TREM-1 mAb (10 $\mu \mathrm{g} / \mathrm{ml}$; clone 193015, R\&D Systems $)$ or an isotype-matched control antibody (10 $\mu \mathrm{g} / \mathrm{ml}$ mouse IgG1, R\&D Systems) for 24 hours. Synovial CD $141^{+}$DC were subsequently stained with a panel of fluorochrome-conjugated antibodies specific for CD80, CD86, and CD40. For T cell assays, CD $141^{+}$DC stimulated for 24 hours with anti-TREM-1 or IgG1 control were subsequently cultured for 5 days with allogeneic $\mathrm{CD}^{+} \mathrm{T}$ cells at a ratio of 1:10. Supernatants were harvested after this time, and the levels of IL-17a and IFN- $\gamma$ (R\&D systems) were measured by ELISA according to manufacturer's instructions.

Statistics. SPSS15 system (SPSS Inc.) for Windows was used for statistical analysis. Wilcoxon Signed Rank test or Mann Whitney $U$ was used for analysis of nonparametric data. Two-tailed student's $t$ test was used for parametric data. $P$ values of less than $0.05\left({ }^{*} P<0.05\right)$ were determined as statistically significant.

Study approval. Ethical approval to conduct this study was granted by St. Vincent's Healthcare Group Medical Research and Ethics Committee and the Tallaght Hospital/St. James's Hospital Joint Research Ethics Committee, and all patients gave fully informed written consent prior to inclusion. All experiments were performed in accordance with these guidelines and regulations.

\section{Author contributions}

MC, AMW, VB, SMW, TM, VM, BM, MB, HC, SW, and UF conducted experiments, analysis of data, and manuscript preparation. UF, DJV, SN, JMF, and MC participated in the data analysis and interpretation and manuscript preparation. UF, DJV, JMF, and MC participated in the study design. UF supervised the research. DJV, RM, and CO recruited all patients and provided all clinical information. All authors read and approved the final manuscript.

\section{Acknowledgments}

This work was supported by the Health Research Board of Ireland, Irish Research Council, and Arthritis Ireland. The authors would like to thank Francis Young, Deborah Cluxton, Sian Cregan, and Gavin McManus for assistance with this study.

Address for correspondence: Ursula Fearon, Department of Molecular Rheumatology, Trinity Biomedical Sciences Institute, Trinity College Dublin, Dublin, Ireland. Phone: 35.312.213436; Email: fearonu@tcd.ie. 
1. Bachem A, et al. Superior antigen cross-presentation and XCR1 expression define human CD11c+CD141+ cells as homologues of mouse CD8+ dendritic cells. J Exp Med. 2010;207(6):1273-1281.

2. Crozat $\mathrm{K}$, et al. The XC chemokine receptor 1 is a conserved selective marker of mammalian cells homologous to mouse CD8alpha+ dendritic cells. J Exp Med. 2010;207(6):1283-1292.

3. Jongbloed SL, et al. Human CD141+ (BDCA-3)+ dendritic cells (DCs) represent a unique myeloid DC subset that cross-presents necrotic cell antigens. J Exp Med. 2010;207(6):1247-1260.

4. Poulin LF, et al. Characterization of human DNGR-1+ BDCA3+ leukocytes as putative equivalents of mouse CD8alpha+ dendritic cells. J Exp Med. 2010;207(6):1261-1271.

5. Haniffa M, et al. Human tissues contain CD141hi cross-presenting dendritic cells with functional homology to mouse CD103+ nonlymphoid dendritic cells. Immunity. 2012;37(1):60-73.

6. Kelly A, et al. CD141+ myeloid dendritic cells are enriched in healthy human liver. J Hepatol. 2014;60(1):135-142.

7. Poulin LF, et al. DNGR-1 is a specific and universal marker of mouse and human Batf3-dependent dendritic cells in lymphoid and nonlymphoid tissues. Blood. 2012;119(25):6052-6062.

8. Watchmaker PB, et al. Comparative transcriptional and functional profiling defines conserved programs of intestinal DC differentiation in humans and mice. Nat Immunol. 2014;15(1):98-108.

9. Pettit AR, MacDonald KP, O’Sullivan B, Thomas R. Differentiated dendritic cells expressing nuclear RelB are predominantly located in rheumatoid synovial tissue perivascular mononuclear cell aggregates. Arthritis Rheum. 2000;43(4):791-800.

10. Page G, Lebecque S, Miossec P. Anatomic localization of immature and mature dendritic cells in an ectopic lymphoid organ: correlation with selective chemokine expression in rheumatoid synovium. J Immunol. 2002;168(10):5333-5341.

11. Jongbloed SL, et al. Enumeration and phenotypical analysis of distinct dendritic cell subsets in psoriatic arthritis and rheumatoid arthritis. Arthritis Res Ther. 2006;8(1):R15.

12. Ng CT, et al. Synovial tissue hypoxia and inflammation in vivo. Ann Rheum Dis. 2010;69(7):1389-1395.

13. Cavanagh LL, et al. Rheumatoid arthritis synovium contains plasmacytoid dendritic cells. Arthritis Res Ther. 2005;7(2):R230-R240.

14. Segura E, et al. Human inflammatory dendritic cells induce Th17 cell differentiation. Immunity. 2013;38(2):336-348.

15. Gao W, McGarry T, Orr C, McCormick J, Veale DJ, Fearon U. Tofacitinib regulates synovial inflammation in psoriatic arthritis, inhibiting STAT activation and induction of negative feedback inhibitors. Ann Rheum Dis. 2016;75(1):311-315.

16. Brutkiewicz RR. Cell Signaling Pathways That Regulate Antigen Presentation. J Immunol. 2016;197(8):2971-2979.

17. Seif F, Khoshmirsafa M, Aazami H, Mohsenzadegan M, Sedighi G, Bahar M. The role of JAK-STAT signaling pathway and its regulators in the fate of T helper cells. Cell Commun Signal. 2017;15(1):23.

18. Engering A, Pieters J. Association of distinct tetraspanins with MHC class II molecules at different subcellular locations in human immature dendritic cells. Int Immunol. 2001;13(2):127-134.

19. Shurin GV, et al. Small rho GTPases regulate antigen presentation in dendritic cells. J Immunol. 2005;174(6):3394-3400

20. de Saint-Vis B, et al. A novel lysosome-associated membrane glycoprotein, DC-LAMP, induced upon DC maturation, is transiently expressed in MHC class II compartment. Immunity. 1998;9(3):325-336.

21. Bánki Z, et al. Cross-linking of CD32 induces maturation of human monocyte-derived dendritic cells via NF-kappa B signaling pathway. J Immunol. 2003;170(8):3963-3970.

22. Crawford $\mathrm{K}$, et al. CD2 engagement induces dendritic cell activation: implications for immune surveillance and T-cell activation. Blood. 2003;102(5):1745-1752.

23. Fallarino F, et al. Murine plasmacytoid dendritic cells initiate the immunosuppressive pathway of tryptophan catabolism in response to CD200 receptor engagement. J Immunol. 2004;173(6):3748-3754.

24. Malinova D, et al. WASp-dependent actin cytoskeleton stability at the dendritic cell immunological synapse is required for extensive, functional T cell contacts. J Leukoc Biol. 2016;99(5):699-710.

25. Al-Alwan MM, Rowden G, Lee TD, West KA. The dendritic cell cytoskeleton is critical for the formation of the immunological synapse. J Immunol. 2001;166(3):1452-1456.

26. Jancic C, et al. Interactions of dendritic cells with fibronectin and endothelial cells. Immunology. 1998;95(2):283-290.

27. Pendl GG, et al. Immature mouse dendritic cells enter inflamed tissue, a process that requires E- and P-selectin, but not P-selectin glycoprotein ligand 1. Blood. 2002;99(3):946-956.

28. Harry RA, Anderson AE, Isaacs JD, Hilkens CM. Generation and characterisation of therapeutic tolerogenic dendritic cells for rheumatoid arthritis. Ann Rheum Dis. 2010;69(11):2042-2050.

29. Burmester GR, et al. A randomised phase IIb study of mavrilimumab, a novel GM-CSF receptor alpha monoclonal antibody, in the treatment of rheumatoid arthritis. Ann Rheum Dis. 2017;76(6):1020-1030.

30. Berner B, Akça D, Jung T, Muller GA, Reuss-Borst MA. Analysis of Th1 and Th2 cytokines expressing CD4+ and CD8+ T cells in rheumatoid arthritis by flow cytometry. J Rheumatol. 2000;27(5):1128-1135.

31. Costello PJ, et al. Psoriatic arthritis joint fluids are characterized by CD8 and CD4 T cell clonal expansions appear antigen driven. J Immunol. 2001;166(4):2878-2886.

32. Menon B, et al. Interleukin-17+CD8+ T Cells Are Enriched in the Joints of Patients With Psoriatic Arthritis and Correlate With Disease Activity and Joint Damage Progression. Arthritis Rheumatol. 2014;66(5):1272-1281.

33. Gregersen PK, Silver J, Winchester RJ. The shared epitope hypothesis. An approach to understanding the molecular genetics of susceptibility to rheumatoid arthritis. Arthritis Rheum. 1987;30(11):1205-1213.

34. Schmidt D, Goronzy JJ, Weyand CM. CD4+ CD7- CD28- T cells are expanded in rheumatoid arthritis and are characterized by autoreactivity. J Clin Invest. 1996;97(9):2027-2037.

35. Yudoh K, Matsuno H, Nakazawa F, Yonezawa T, Kimura T. Reduced expression of the regulatory CD4+ T cell subset is related to Th1/Th2 balance and disease severity in rheumatoid arthritis. Arthritis Rheum. 2000;43(3):617-627.

36. Rao DA, et al. Pathologically expanded peripheral T helper cell subset drives B cells in rheumatoid arthritis. Nature. 2017;542(7639):110-114.

37. Basdeo SA, et al. Polyfunctional, Pathogenic CD161+ Th17 Lineage Cells Are Resistant to Regulatory T Cell-Mediated Suppression in the Context of Autoimmunity. J Immunol. 2015;195(2):528-540. 
38. Tullett KM, et al. Targeting CLEC9A delivers antigen to human CD141+ DC for CD4+ and CD8+T cell recognition. JCI Insight. 2016;1(7):e87102.

39. Sponaas AM, et al. PDL1 Expression on Plasma and Dendritic Cells in Myeloma Bone Marrow Suggests Benefit of Targeted anti PD1-PDL1 Therapy. PLoS ONE. 2015;10(10):e0139867.

40. Ahrens S, et al. F-actin is an evolutionarily conserved damage-associated molecular pattern recognized by DNGR-1, a receptor for dead cells. Immunity. 2012;36(4):635-645

41. Nair A, et al. Synovial chemokine expression and relationship with knee symptoms in patients with meniscal tears. Osteoarthr Cartil. 2015;23(7):1158-1164.

42. Müller-Ladner U, et al. Synovial fibroblasts of patients with rheumatoid arthritis attach to and invade normal human cartilage when engrafted into SCID mice. Am J Pathol. 1996;149(5):1607-1615.

43. Lefèvre S, et al. Synovial fibroblasts spread rheumatoid arthritis to unaffected joints. Nat Med. 2009;15(12):1414-1420

44. Gao W, McCormick J, Connolly M, Balogh E, Veale DJ, Fearon U. Hypoxia and STAT3 signalling interactions regulate pro-inflammatory pathways in rheumatoid arthritis. Ann Rheum Dis. 2015;74(6):1275-1283.

45. Moran EM, et al. IL-17A expression is localised to both mononuclear and polymorphonuclear synovial cell infiltrates. PLoS One. 2011;6(8):e24048

46. Biniecka M, et al. Dysregulated bioenergetics: a key regulator of joint inflammation. Ann Rheum Dis. 2016;75(12):2192-2200.

47. Bosco MC, et al. Hypoxia modulates the gene expression profile of immunoregulatory receptors in human mature dendritic cells: identification of TREM-1 as a novel hypoxic marker in vitro and in vivo. Blood. 2011;117(9):2625-2639.

48. Verdijk P, et al. Morphological changes during dendritic cell maturation correlate with cofilin activation and translocation to the cell membrane. Eur J Immunol. 2004;34(1):156-164.

49. Andersson U, Erlandsson-Harris H. HMGB1 is a potent trigger of arthritis. J Intern Med. 2004;255(3):344-350.

50. Veale DJ, et al. Intra-articular primatised anti-CD4: efficacy in resistant rheumatoid knees. A study of combined arthroscopy, magnetic resonance imaging, and histology. Ann Rheum Dis. 1999;58(6):342-349.

51. Li B, Ruotti V, Stewart RM, Thomson JA, Dewey CN. RNA-Seq gene expression estimation with read mapping uncertainty. Bioinformatics. 2010;26(4):493-500.

52. Ritchie ME, et al. limma powers differential expression analyses for RNA-sequencing and microarray studies. Nucleic Acids Res 2015;43(7):e47.

53. Law CW, Chen Y, Shi W, Smyth GK. voom: Precision weights unlock linear model analysis tools for RNA-seq read counts. Genome Biol. 2014;15(2):R29.

54. Kennedy A, et al. Tumor necrosis factor blocking therapy alters joint inflammation and hypoxia. Arthritis Rheum. 2011;63(4):923-932. 